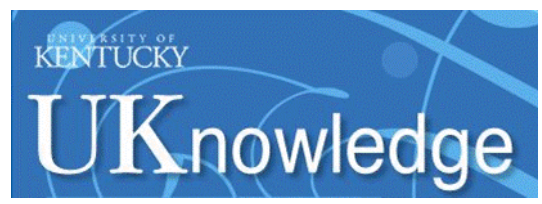

University of Kentucky

UKnowledge

Physics and Astronomy Faculty Publications

Physics and Astronomy

7-23-2015

\title{
A Critical Assessment of Stellar Mass Measurement Methods
}

\author{
Bahram Mobasher \\ University of California - Riverside \\ Tomas Dahlen \\ Space Telescope Science Institute \\ Henry C. Ferguson \\ Space Telescope Science Institute \\ Viviana Acquaviva \\ CUNY NYC College of Technology \\ Guillermo Barro \\ University of California - Santa Cruz \\ Follow this and additional works at: https://uknowledge.uky.edu/physastron_facpub

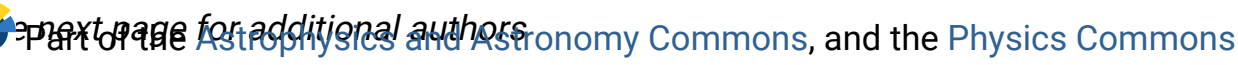

Right click to open a feedback form in a new tab to let us know how this document benefits you.

\section{Repository Citation}

Mobasher, Bahram; Dahlen, Tomas; Ferguson, Henry C.; Acquaviva, Viviana; Barro, Guillermo; Finkelstein, Steven L.; Fontana, Adriano; Gruetzbauch, Ruth; Johnson, Seth; Lu, Yu; Papovich, Casey; Pforr, Janine; Salvato, Mara; Somerville, Rachel S.; Wiklind, Tommy; Wuyts, Stijn; Ashby, Matthew L.N.; Bell, Eric; Conselice, Christopher J.; Dickinson, Mark E.; Faber, Sandra M.; Fazio, Giovanni; Finlator, Kristian; Galametz, Audrey; Gawiser, Eric; Giavalisco, Mauro; Grazian, Andrea; Grogin, Norman A.; Guo, Yicheng; Hathi, Nimish; Kocevski, Dale; Koekemoer, Anton M.; Koo, David C.; Newman, Jeffrey A.; Reddy, Naveen; Santini, Paola; and Wechsler, Risa H., "A Critical Assessment of Stellar Mass Measurement Methods" (2015). Physics and Astronomy Faculty Publications. 254.

https://uknowledge.uky.edu/physastron_facpub/254

This Article is brought to you for free and open access by the Physics and Astronomy at UKnowledge. It has been accepted for inclusion in Physics and Astronomy Faculty Publications by an authorized administrator of UKnowledge. For more information, please contact UKnowledge@lsv.uky.edu. 


\section{A Critical Assessment of Stellar Mass Measurement Methods}

\section{Digital Object Identifier (DOI)}

http://dx.doi.org/10.1088/0004-637X/808/1/101

\section{Notes/Citation Information}

Published in The Astrophysical Journal, v. 808, no. 1, article 101, p. 1-28.

(c) 2015. The American Astronomical Society. All rights reserved.

Reproduced by permission of the AAS.

\section{Authors}

Bahram Mobasher, Tomas Dahlen, Henry C. Ferguson, Viviana Acquaviva, Guillermo Barro, Steven L. Finkelstein, Adriano Fontana, Ruth Gruetzbauch, Seth Johnson, Yu Lu, Casey Papovich, Janine Pforr, Mara Salvato, Rachel S. Somerville, Tommy Wiklind, Stijn Wuyts, Matthew L.N. Ashby, Eric Bell, Christopher J. Conselice, Mark E. Dickinson, Sandra M. Faber, Giovanni Fazio, Kristian Finlator, Audrey Galametz, Eric Gawiser, Mauro Giavalisco, Andrea Grazian, Norman A. Grogin, Yicheng Guo, Nimish Hathi, Dale Kocevski, Anton M. Koekemoer, David C. Koo, Jeffrey A. Newman, Naveen Reddy, Paola Santini, and Risa H. Wechsler 


\title{
A CRITICAL ASSESSMENT OF STELLAR MASS MEASUREMENT METHODS
}

\author{
Bahram Mobasher $^{1}$, Tomas Dahlen ${ }^{2}$, Henry C. Ferguson $^{2}$, Viviana Acquaviva ${ }^{3}$, Guillermo Barro ${ }^{4}$, \\ Steven L. Finkelstein ${ }^{5}$, Adriano Fontana ${ }^{6}$, Ruth Gruetzbauch ${ }^{7}$, Seth Johnson ${ }^{8}$, Yu Lu ${ }^{9}$, Casey J. Papovich $^{10}$, \\ Janine Pforr $^{11}$, Mara Salvato ${ }^{12}$, Rachel S. Somerville ${ }^{13}$, Tommy Wiklind ${ }^{14}$, Stijn Wuyts ${ }^{12}$, Matthew L. N. Ashby ${ }^{15}$, \\ Eric Bell ${ }^{16}$, Christopher J. Conselice ${ }^{17}$, Mark E. Dickinson ${ }^{11}$, SAndra M. Faber ${ }^{4}$, Giovanni Fazio ${ }^{15}$, \\ Kristian Finlator $^{18}$, Audrey Galametz ${ }^{6}$, Eric Gawiser ${ }^{13}$, Mauro Giavalisco ${ }^{8}$, Andrea Grazian ${ }^{6}$, Norman A. Grogin ${ }^{2}$, \\ Yicheng Guo ${ }^{4}$, Nimish Hathi ${ }^{19}$, Dale Kocevski ${ }^{20}$, Anton M. Koekemoer ${ }^{2}$, David C. Koo ${ }^{4}$, Jeffrey A. Newman ${ }^{21}$, \\ Naveen Reddy ${ }^{1}$, PaOla Santini ${ }^{6}$, and Risa H. WeChSler ${ }^{9}$ \\ ${ }^{1}$ Department of Physics and Astronomy, University of California, Riverside, CA 92521, USA \\ ${ }^{2}$ Space Telescope Science Institute, 3700 San Martin Drive, Baltimore, MD 21218, USA \\ ${ }^{3}$ Physics Department, CUNY NYC College of Technology, 300 Jay Street, Brooklyn, NY 11201, USA \\ ${ }^{4}$ UCO/Lick Observatory, Department of Astronomy and Astrophysics, University of California, Santa Cruz, CA 95064, USA \\ ${ }_{6}^{5}$ Department of Astronomy, The University of Texas at Austin, Austin, TX 78712, USA \\ ${ }^{6}$ INAF, Osservatorio Astronomico di Roma, Via Frascati 33, I-00040, Monteporzio, Italy \\ ${ }^{7}$ Center for Astronomy and Astrophysics, Observatorio Astronomico de Lisboa, Tapada da Ajuda, 1349-018, Lisboa, Portugal \\ 8 Department of Astronomy, University of Massachusetts, 710 North Plesant Street, Amherst, MA 01003, USA \\ ${ }^{9}$ Kavli Institute for Particle Physics and Cosmology, Stanford University, Stanford, CA 94305, USA \\ ${ }^{10}$ Department of Physics and astronomy, Texas A\&M Research Foundation, College Station, TX 77843, USA \\ ${ }^{11}$ National Optical Astronomy Observatories, $950 \mathrm{~N}$ Cherry Avenue, Tucson, AZ 85719, USA
${ }^{12}$ Max-Planck-Institut für extraterrestrische Physik, Giessenbachstrasse 1, D-85748 Garching bei München, Germany \\ ${ }^{13}$ Department of Physics and Astronomy, Rutgers, The State University of New Jersey, \\ 136 Frelinghuysen Road, Piscataway, NJ 08854, USA \\ ${ }^{14}$ Joint ALMA Observatory, Alonso de Cordova 3107, Vitacura, Santiago, Chile \\ ${ }^{15}$ Harvard Smithsonian Center for Astrophysics, 60 Garden Street, MS-66, Cambridge, MA 02138-1516, USA \\ ${ }_{16}$ Department of Astronomy, University of Michigan, 500 Church Street, Ann Arbor, MI 48109, USA \\ ${ }^{17}$ School of Physics and Astronomy, University of Nottingham, Nottingham, UK \\ ${ }^{18}$ Dark Cosmology Center, Niels Bohr Institute, University of Copenhagen, Denmark \\ ${ }^{19}$ LAM-Laboratoire d'Astrophysique de Marseille, 38, Rue R. Jollot-Curie, F-13388 Marseille, Cedex 13, France \\ ${ }_{21}^{20}$ Department of Physics and Astronomy, University of Kentucky, Lexington, KY 40506, USA \\ 21 Department of Physics and Astronomy, University of Pittsburgh, Pittsburgh, PA 15260, USA \\ Received 2014 October 11; accepted 2015 May 4; published 2015 July 23
}

\begin{abstract}
This is the second paper in a series aimed at investigating the main sources of uncertainty in measuring the observable parameters in galaxies from their spectral energy distributions (SEDs). In the first paper we presented a detailed account of the photometric redshift measurements and an error analysis of this process. In this paper we perform a comprehensive study of the main sources of random and systematic error in stellar mass estimates for galaxies, and their relative contributions to the associated error budget. Since there is no prior knowledge of the stellar mass of galaxies (unlike their photometric redshifts), we use mock galaxy catalogs with simulated multiwaveband photometry and known redshift, stellar mass, age and extinction for individual galaxies. The multiwaveband photometry for the simulated galaxies were generated in 13 filters spanning from $U$-band to mid-infrared wavelengths. Given different parameters affecting stellar mass measurement (photometric signal-to-noise ratios $(\mathrm{S} / \mathrm{N})$, SED fitting errors and systematic effects), the inherent degeneracies and correlated errors, we formulated different simulated galaxy catalogs to quantify these effects individually. For comparison, we also generated catalogs based on observed photometric data of real galaxies in the Great Observatories Origins Deep SurveySouth field, spanning the same passbands. The simulated and observed catalogs were provided to a number of teams within the Cosmic Assembly Near-infrared Deep Extragalactic Legacy Survey collaboration to estimate the stellar masses for individual galaxies. A total of 11 teams participated, with different combinations of stellar mass measurement codes/methods, population synthesis models, star formation histories, extinction and age. For each simulated galaxy, the differences between the input stellar masses, $M_{\text {input }}$, and those estimated by each team, $M_{\text {est }}$, is defined as $\Delta \log (M) \equiv \log \left(M_{\text {estimated }}\right)-\log \left(M_{\text {input }}\right)$, and used to identify the most fundamental parameters affecting stellar mass estimate in galaxies, with the following results. (1) No significant bias in $\Delta \log (M)$ was found among different codes, with all having comparable scatter $(\sigma(\Delta \log (M))=0.136$ dex $)$. The estimated stellar mass values are seriously affected by low photometric $\mathrm{S} / \mathrm{N}$, with the rms scatter increasing for galaxies with $H_{\mathrm{AB}}>26 \mathrm{mag}$; (2) A source of error contributing to the scatter in $\Delta \log (M)$ is found to be due to photometric uncertainties $(0.136 \mathrm{dex})$ and low resolution in age and extinction grids when generating the SED templates; (3) The median of stellar masses among different methods provides a stable measure of the mass associated with any given galaxy $(\sigma(\Delta \log (M))=0.142$ dex $)$; (4) The $\Delta \log (M)$ values are strongly correlated with deviations in age (defined as the difference between the estimated and expected values), with a weaker correlation with extinction; (5) The rms scatter in the estimated stellar masses due to free parameters (after fixing redshifts and initial mass function) are quantified and found to be $\sigma(\Delta \log (M))=0.110 \mathrm{dex}$; (6) Using the observed data, we studied the
\end{abstract}


sensitivity of stellar masses to both the population synthesis codes and inclusion of nebular emission lines and found them to affect the stellar mass by 0.2 and 0.3 dex respectively.

Key words: galaxies: distances and redshifts - galaxies: high-redshift - galaxies: photometry - surveys

\section{INTRODUCTION}

The questions of what governs the observed properties of galaxies, the reason behind the correlations among these properties and how they change with look-back time, are among the most fundamental in observational astronomy today. This requires accurate measurement of redshifts as well as the rest-frame observables. In particular, detailed knowledge of the statistical properties of galaxies (i.e., luminosity and mass functions) at different redshifts is essential to constrain current hierarchical models for the formation of galaxies. This requires large and deep surveys with multi-waveband photometry, photometric redshifts and stellar mass estimates.

The installation of wide field of view detectors with high optical and infrared quantum efficiency on space and groundbased observatories has now allowed construction of multiwaveband, large and deep galaxy surveys. These surveys occupy a large portion of the area-depth parameter space, from the very deep Hubble Ultra Deep Field (Beckwith et al. 2006), designed for studies of very high redshift galaxies, to wide-area Cosmic Evolution Survey-COSMOS (Scoville et al. 2007) formulated to study the large scale structure in the universe and its evolution with redshift. These are complemented by the intermediate surveys (in terms of depth and area) such as the Great Observatories Origins Deep Survey (GOODS; Giavalisco et al. 2004), designed specifically for studies of the evolution of galaxies to high redshifts. Recently, the wavelength range of these surveys has been extended to nearinfrared bands in a Multi-cycle Treasury program, the Cosmic Assembly Near-infrared Deep Extragalactic Legacy Survey (CANDELS) (Grogin et al. 2011; Koekemoer et al. 2011). One important addition to these observations is the availability of deep mid-infrared data $(3.6-8.0 \mu \mathrm{m})$ from the Spitzer Space Telescope, extending the observed wavelength range to $8 \mu \mathrm{m}$ (Ashby et al. 2013). This is essential in constraining the spectral energy distributions (SEDs) of galaxies and in estimating accurate photometric redshifts and stellar masses spanning a range of redshifts.

The multi-waveband data from these surveys are extensively used to study the luminosity function and mass function of galaxies to very high redshifts, with often divergent results (Ouchi et al. 2009; Bouwens et al. 2011; Finkelstein et al. 2012; Dahlen et al. 2013; McLure et al. 2013; Schenker et al. 2013b). This is done through fitting of the observed SEDs of individual galaxies to model templates in order to estimate their photometric redshifts or measure rest-frame luminosities. However, there are a number of concerns regarding this process. First, this requires accurate photometry for galaxies. Given that the photometric data points used for the SED fits are observed by different telescopes and instruments, with different point spread functions (PSFs), one needs to reduce them to the same scale (i.e., images with the highest resolution). This is to ensure that they are corrected so that the ratios of fluxes in different bands refer to the same regions of galaxies. Second, this requires clear understanding of the accuracy and biases in photometric redshift and stellar mass measurement. Third, at the basic level, different investigators use different techniques, codes, templates and initial parameters to fit the observed SEDs and extract observable information from them. This alone introduces unknown differences in the photometric redshift and stellar mass estimates to the same galaxy. The first problem is generally addressed by degrading the data to a common PSF, or by fitting templates for galaxies from the higher resolution image convolved with a kernel to match the PSF of the lower resolution images, using the Template Fitting (TFIT) technique (Laidler et al. 2007). This has successfully been used to generate self-consistent multiband dataset for individual galaxies across the wavelength range covered (Guo et al. 2013; H. Nayyeri et al. 2015, in preparation; Santini et al. 2015). However, there are still outstanding issues regarding the second and third points. In Dahlen et al. (2013), we addressed systematic uncertainties in photometric redshift estimation. In this paper, we focus on the stellar mass measurement.

The most common method for measuring the stellar masses of galaxies is to fit their observed SEDs, covering the wavelength range UV/optical/infrared, to templates generated from the population synthesis models. The templates consist of a large grid of model SEDs with a range of free parameters, including: redshift, SFH, age, prescription for dust extinction and metallicity. For any galaxy, the parameters corresponding to the template SED which best fit its observed SED (minimum $\left.\chi^{2}\right)$ are associated to that galaxy. Having measured the massto-light ratio $(M / L)$ of the galaxy, and knowing its absolute luminosity, one could then estimate its stellar mass, as well as other physical parameters. However, there is significant degeneracy in this procedure. The fitting techniques do not necessarily yield unique models, with various combinations of free parameters leading to equally acceptable fits. Furthermore, the final estimate of the stellar mass also depends on technical details such as the population synthesis models used to generate template SEDs, the fitting technique, the code used and the signal-to-noise ratio $(\mathrm{S} / \mathrm{N})$ of the observed photometric data. Therefore, it is important to understand the dependence of the stellar mass on each of these parameters and to disentangle the interplay between them. With this in mind, we have undertaken an extensive investigation of the sources of uncertainty in the stellar mass measurement from broadband photometry. The time is ripe for such a study, with the availability of the CANDELS data spanning a wide range in wavelength.

We perform two classes of tests: (1) comparison of estimated stellar masses with "true" ones generated from theoretical mock catalogs and (2) comparison of estimated stellar masses from different codes and methods applied to observational data, where the "true" masses are not known. This allows a test of internal consistency between different stellar mass methods, aiming to understand sources responsible for the observed divergencies between them. The CANDELS data used for this purpose are extremely deep, so the photometry has very low measurement errors.

We generated simulated and real multi-waveband photometric catalogs of galaxies with known redshifts and stellar masses and asked a number of experts within the CANDELS team to independently estimate the observable quantities 
associated with them. We then compared the stellar masses with the "true" values in the mock catalogs and the measurements between different teams, aiming first to have a realistic estimate of the error budget and then to develop a prescription to acquire the most accurate stellar mass for individual galaxies. Furthermore, we aim to understand parameters responsible for the observed divergencies between different algorithms used for stellar mass measurement.

Several studies have recently undertaken similar investigation by addressing the accuracy of predicted physical parameters in galaxies using simulated catalogs (Wuyts et al. 2009; Lee et al. 2012; Pforr et al. 2012). These studies often used one population synthesis codes to generate model templates (Wuyts et al. 2009) and a single SED fitting technique (Longhetti \& Saracco 2009). Furthermore, in the fitting process they fit all the free parameters simultaneously (i.e., age, metallicity, SFH, mass), causing serious degeneracies between the predicted parameters. Moreover, they either use a limited redshift range (Wuyts et al. 2009) or are restricted to certain galaxy types (Lee et al. 2012) and are hardly constrained by the observational data (Pforr et al. 2012). Pforr et al. (2013) investigated the dependence of results on different population models, used a wider range in redshift, and explored the depndence of results on wavelength coverage and photometric filters. While they used Maraston (2005) as their population synthesis model, they also tested the results from other codes but used the same SED fitting method and procedure to estimate the parameters, showing serious degeneracies. None of these studies explores the dependence of the estimated stellar masses on the nebular emission lines, which is proved to be significant (de Barros et al. 2014).

This paper complements previous studies in various ways. It uses 10 independent SED fitting techniques and codes from different teams and, at the same time, explores dependence of each of these results on a variety of population synthesis models. Furthermore, the mock catalogs generated for this purpose are selected to resemble observed galaxy surveys (i.e., CANDELS) in terms of redshift distribution, wavelength coverage and photometric uncertainty, so that the results would be directly applicable to the observed data. In addition to simulations, it also uses observed photometry and real data to explore the internal consistency of the stellar masses measured from different procedures. By fixing the parameters in the SED fitting process to those of the input mock catalogs, we study the degeneracy among the parameters, estimating the errors contributed from each parameter to the final stellar mass. The present study also investigates dependence of stellar mass on nebular line emissions. Finally, the errors associated with individual physical parameters are estimated and their contribution to the total error budget calculated. Results from this study are directly used to estimate stellar masses for the CANDELS galaxies by finding the technique which leads to the most accurate measurement.

In Section 2, we present the procedures and the tests designed for this study. In Section 3 the participating teams are introduced, with a brief description of the methods and techniques used by each team. Sections 4-7 present different tests and explore sources of uncertainty and bias in stellar mass measurements from different teams. Comparison with other similar studies in literature is performed in Section 8, with the error budgests estimated and discussed in Section 9.
Table 1

Details of different TESTs Developed for Stellar Mass Measurement

TEST-1:

Fixed Parameters

IMF: Chabrier (limits: $0.1<M / M_{\odot}<100$ )

Redshift range $0<z<4$

Stellar population templates: Bruzual \& Charlot (2003) (BC03).

SFH: Single burst Exponentially declining, $\tau$ fixed at $0.1 \mathrm{Gyr}$. Gas recycling: no.

Dust extinction law: Calzetti.

IGM Absorption: Madau law, flux set to zero at $\lambda<912 \AA$ (restframe).

Metallicity: $Z=Z$.

Nebular Emission: not included.

Free Parameters

Age between $10 \mathrm{Myr}$ and the Age of the universe at the redshift of the galaxy.

$E(B-V)$ between 0 and 1 .

TEST-2A

Fixed parameters

Chabrier IMF $\left(0.1<M / M_{\odot}<100\right)$

Redshift range $0<z<6$

Redshift is fixed to its input value.

Dust extinction is applied to the photometry in the mock catalog.

Free parameters

${ }^{\text {a }} \mathrm{SFH}$, metallicity, extinction, population synthesis code

TEST-2B

Fixed Parameters

Templates: BC03 with Chabrier IMF $\left(0.1<M / M_{\odot}<100\right)$

Redshift range $0<z<6$

${ }^{\text {a }} \mathrm{SFH}$ : exponentially declining SFR

no extinction applied to the photometric points; $E(B-V)$ $=0$

Metallicity: solar

Emission lines: not included

Redshift fixed to the provided value in the mock catalog

Free parameters

The exponential time scale $\tau$ and the age of the stellar population.

TEST-3A

Fixed Parameters

Observed F160W band selected multi-band TFIT photometric catalog for GOODS-S

The objects are fixed at their spectroscopic redshifts

Redshift range $0<z<6$

Chabrier IMF $\left(0.1<M / M_{\odot}<100\right)$

Free Parameters

SFH, metallicity, extinction, population synthesis code, stellar mass, age

TEST-3B

Fixed Parameters

Observed F160W band selected multi-band TFIT photometric catalog for GOODS-S

Templates: BC03 [with Chabrier IMF $\left(0.1<M / M_{\odot}<100\right)$ ]

Extinction: $E(B-V)=\mathrm{Av}=0$, i.e., no extinction

${ }^{\text {a }} \mathrm{SFH}$ : Exponentially declining

Metallicity: Solar

Free Parameters

Redshift range $0<z<6$

TEST-4

Stellar mass, star formation time-scale, $\tau$, age

The same as TEST-3A but selected in ACS $z$-band, with shallower observed near-infrared data

Notes. The listed parameters are used to generate the mock catalogs and as inputs in the codes discussed in Section 3 to measure stellar masses.

${ }^{a}$ The SAMs use a diversity of SFHs depending on the host halo merger history. Therefore, the SFH of every mock galaxy is fixed. The forms of the SFHs adapted here are used to generate the SED templates for the stellar mass measurement methods. 
Our conclusions are summarized in Section 10. Throughout, we assume standard cosmology with $H_{0}=70 \mathrm{Km} \mathrm{s}^{-1} \mathrm{Mpc}^{-1}$, $\Omega_{M}=0.3$ and $\Omega_{\Lambda}=0.7$. Magnitudes are all in the AB system (Oke \& Gunn 1983).

\section{THE PROCEDURE}

In this investigation, we perform four different tests, designed to explore different types of systematic errors in stellar mass measurement. We estimate stellar masses from different catalogs: an empirical mock catalog (TEST-1), a Semi-Analytic Mock catalog (SAM; TEST-2) and a "real" observational catalog (TEST-3 and TEST-4). The main parameters used to generate the mock catalogs and the input to stellar mass measurement codes (discussed in Section 3) are listed in Table 1. In Appendix A, we summarize definitions of the stellar masses used in the SAMs in this study and most commonly used in literature. TEST-1, developed to evaluate different SED fitting codes, fits simulated data for galaxies with simple SFHs, using a limited number of free parameters (this test is strongly constrained). The mock catalogs are generated to have similar distribution of physical parameters as the observed catalogs (presented in Appendix B). TEST-2A and TEST-2B fit simulated data for galaxies with more complex SFHs drawn from a semi-analytic model. TEST-2A fits the mock data, simulated to mimic real galaxies as closely as possible. TEST-2B is more constrained; there is no dust and fits are restricted to using the same evolutionary synthesis code for the fits. TEST-3A and TEST-3B compare masses when the same fitting parameters and techniques, used in TEST-2A and TEST-2B, are applied to real galaxies. TEST-4 repeats TEST3A using somewhat shallower near-IR data typical of preCANDELS observations. The simulated multi-waveband mock catalogs used in TEST-2A and 2B were generated with halos extracted from Bolshoi $N$-body simulations (Klypin et al. 2011; Behroozi et al. 2013) and populated using semi-analytic models (Somerville et al. 2008, 2012). The bandpasses and quality of the photometry in all the tests approximate the observed data from the CANDELS. The stellar masses provided in the mock catalogs are defined as the mass which is directly produced through SED fits. The age is defined as the time since the on-set of star formation. One of the main sources of error in stellar mass estimates is lack of knowledge of the SFHs (e.g., Lee et al. 2015). Nearly all the methods make very simple assumptions about this and even when diverse SFHs are allowed, it is unclear whether the methods can correctly select the right type of history based on the photometry, given all the other uncertainties. The SAMs have a semi-realistic mix of complex SFHs (including rising, constant, and declining) however, they do not correctly reproduce the observed trends between galaxy mass and star formation rate (SFR) i.e., downsizing. The main characteristics of the tests in this section are listed in Table 1, with an overall comparison between different tests presented in Table 2 and detailed below:

TEST-1: Test of the consistency of different SED fitting codes and techniques. This test is designed to study how well different codes can measure the stellar masses and if there is any difference originating from the codes once we keep all the rest of the parameters fixed. To do this, we generate a mock catalog with known input parameters (redshift, stellar mass, age and extinction) using templates produced from Bruzual \& Charlot (2003) population synthesis models. To make the simulated galaxies comparable to the real data, we add noise to their photometry. The parameters used to generate the mock SEDs are listed in Table 1. There are a total of 559 galaxies in TEST-1 mock catalog. The total number of simulated galaxies in TEST-1, and the distribution of their physical parameters are taken to be close to the real spectroscopic catalog in GOODS-S field, used to calibrate photometric redshifts and the SED fitting techniques. This allows results from TEST-1 to be directly applicable to observations. Details about the TEST-1 mock catalog and distribution of the observable parameters are given in Appendix B.

The masses are derived by fixing the template SEDs to have the input values (listed in Table 1) and ONLY fitting for two quantities: the age of the star formation and color excess $(E(B-V))$. The age is defined as the time since the on-set of star formation (assuming an exponentially declining SFR with a fixed $\tau$ ) and was constrained between $10 \mathrm{Myr}$ and the age of the universe at the redshift of the particular galaxy under consideration. The allowed range for the color excess, $E(B-V)$, is taken to be between 0 and 1 . The redshift for each galaxy was fixed to its input value. Since the majority of the parameters affecting the stellar mass measurement are fixed, the only difference between the estimated stellar masses from the SED fits $\left(M_{\mathrm{est}} / M_{\odot}\right)$ and the expected stellar masses $\left(M_{\text {input }} / M_{\odot}\right)$ is due to differences between the codes and the SED fitting techniques used between different teams.

TEST-1 is based on a set of 13 filters consisting of: $U$-band (VIMOS), optical-F435W, F606W, F775W, F850LP (ACS); near-infrared $F 105 W, F 125 W, F 160 W$ (WFC3); HawkI $K$-band (Very Large Telescope (VLT)) and Spitzer/IRAC 3.6, 4.5, 5.8 and $8 \mu \mathrm{m}$. The selection criteria for galaxies in TEST-1 include: (a) $\mathrm{S} / \mathrm{N}>5$ in the $H$-band (the selection band in the simulated catalog); (b) detected with $\mathrm{S} / \mathrm{N}>1$ in at least six passbands: (c) $0<z<4$.

TEST-2: Test of the sensitivity of the stellar mass estimates to the free parameters. This test is developed to study the effect of free parameters on the stellar mass measurement. It uses SAM catalogs containing 10,000 galaxies with known multi-waveband photometry, input mass, age, extinction and metallicity. The SEDs were constructed using Bruzual \& Charlot (2003, BC03) models, with a modified version of Charlot \& Fall (2000) prescription for dust treatment as discussed in Somerville et al. (2012). Stellar mass and chemical evolution are calculated assuming instantaneous recycling. The SFHs are diverse, consisting of exponentially declining, constant and rising. Redshift distribution for galaxies in TEST-2 catalog closely follow the photometric redshift distribution in the GOODS-S field (Appendix B).

For all the galaxies in TEST-2 mock catalogs, photometry is provided in 13 filters: $U$-band (VIMOS), optical- $F 435 W$, $F 606 W, F 775 W, F 850 L P$ (ACS); near-infrared $F 105 W$, F125W, F160W (WFC3); HawkI $K$-band (VLT) and Spitzerl IRAC 3.6, 4.5, 5.8 and $8 \mu \mathrm{m}$. The selection criteria for the mock catalog here are: (a) $\mathrm{S} / \mathrm{N}>5$ in the $H$-band (the selection band in the simulated catalog); (b) Detected with $\mathrm{S} / \mathrm{N}>1$ in at least six passbands; (c) $z<6$. TEST-2 is performed in two stages:

TEST-2A. The mock catalog here is generated using a diversity of SFHs (exponentially declining, rising and constant) and metallicities. The data generated for this catalog have dust extinction applied to the photometry and hence, closely resemble the observations. To estimate the stellar masses, the participating teams were not restricted and were 
Table 2

List of the Parameters in the SED Fitting Methods Which are Kept Fixed to Values Listed in Table 1 or are Left Free in the Fit

\begin{tabular}{|c|c|c|c|c|c|c|}
\hline Parameters & TEST-1 & TEST-2A & TEST-2B & TEST-3A & TEST-3B & TEST-4 \\
\hline Star Formation History & Exp. Declining & Free & Exp. Declining & Free & Exp. Declining & Free \\
\hline Population Synthesis Models & $\mathrm{BC} 03$ & Free & $\mathrm{BC} 03$ & Free & $\mathrm{BC} 03$ & Free \\
\hline$\tau$ & Fixed & Free & Fixed & Free & Fixed & Free \\
\hline $\mathrm{IMF}$ & Fixed & Fixed & Fixed & Fixed & Fixed & Fixed \\
\hline Redshift & Fixed & Fixed & Fixed & Fixed & Fixed & Fixed \\
\hline Extinction & Fixed & Free & None & Free & None & Free \\
\hline Age & Free & Free & Free & Free & Free & Free \\
\hline Metallicity & Fixed & Free & Fixed & Free & Fixed & Free \\
\hline Nebular Emission & No & No & No & No & No & No \\
\hline IGM Absorption & Fixed & Fixed & Fixed & Fixed & Fixed & Fixed \\
\hline
\end{tabular}

free to choose any template from any stellar population synthesis code, SFH, metallicity and extinction law to fit the mock SEDs. The only limitation was to use Chabrier IMF and to fix the redshift of the galaxy to its input value in the mock catalog.

TEST-2B. Unlike TEST-2A, the mock catalog here is generated by constraining the free parameters. The SFH associated with template SEDs is fixed to an exponentially declining form, with the templates produced from BC03 with solar metallicity. No dust extinction is applied to photometry in the mock catalog and therefore, TEST-2B is not representative of the "real" population of galaxies. Redshift is fixed to its input value and Chabrier IMF is used. The participating teams were asked to use the same input parameters as the ones used to generate the mock data.

Comparison between the stellar mass estimates from TEST2A and TEST-2B will therefore reveal the effect of free parameters and degeneracy in the SED fitting process.

TEST-3: Comparison of the stellar mass measurements using real data. Having estimated the sources of scatter in stellar mass measurements due to different codes (TEST-1) and due to degeneracy and interplay between the free parameters (TEST-2), we now apply the methods on a sample of observed SEDs with accurate multi-waveband data and available spectroscopic redshifts, selected from the TFIT catalog in the GOODS-S field (Guo et al. 2013). This is the same sample used in Dahlen et al. (2013) to calibrate the templates for measuring photometric redshifts. A total of 598 galaxies were used for this test. For the SED fits, the galaxy redshifts were fixed to their spectroscopic values. Unlike TEST-1 and TEST2, where we used simulated photometric catalogs and hence, had estimates of the "true" stellar mass, here we do not have any absolute measure of the stellar mass and the comparison is only relative, measuring the consistency between different approaches.

The photometry for TEST-3 is performed on the real data using the TFIT technique (Guo et al. 2013) and consists of: $U$-band (VIMOS), optical-F435W, F606W, F775W, F850LP (ACS); near-infrared-F098M, F105W, F125W, F160W (WFC3); Ks (VLT/ISAAC) and mid-infrared Spitzer/IRAC $3.6,4.5,5.8$ and $8 \mu \mathrm{m}$. The $F 098 M$ is only available for the Early Release Survey part of the GOODS-S, while F105W is only available for a sub-area of that field. TEST-3 is also done in two stages:

$T E S T-3 A$. In this test no restriction was imposed on the free parameters when generating the template SEDs for the fits, except for the redshifts which were fixed to their spectroscopic values and the IMF which was chosen to be Chabrier (Table 1).
The stellar masses were subsequently estimated from different methods (Section 3) and compared with each other.

TEST-3B. This is the same as TEST-3A with additional restrictions imposed on the free parameters. This will show how close the results from different teams would be when some of the parameters in the SED fits are not allowed to vary. Therefore, it indicates the effect of the free parameters (and their possible interplay) in stellar mass measurements.

TEST-4: Tests the effect of selection wavelength and nearinfrared photometric depth on the stellar mass measurement. This is similar to TEST-3A with the only difference being the use of much shallower near-infrared data and selection in ACS $z$-band. This test is designed to examine the way different codes treat shallow infrared data and its effect on stellar mass measurement. As is often the case in galaxy surveys, due to smaller size of near-IR detectors, their lower sensitivity and the effect of sky brightness, the near-IR data are not as deep as their optical counterparts. We designed TEST-4 to examine the sensitivity of stellar mass on these parameters.

\section{THE SED FITTING TECHNIQUES AND STELLAR MASS MEASUREMENT}

The catalogs discussed in Section 2 were provided to the CANDELS team members. Using the instructions for different tests (Table 1), the teams were asked to predict the stellar masses for galaxies in the catalogs, satisfying the requirements for each TEST. To perform this as objectively as possible, the $M_{\text {input }}$ values in the mock catalogs were not revealed to the participants.

A total of 10 teams participated in this exercise (not all the teams participated in all the tests). In many cases, the codes and templates used to measure the stellar masses were different from those used for the photometric redshifts in Dahlen et al. (2013). Below, details of the codes and the assumptions when applied on TESTs 2A, 3A and 4 are described (in these tests the participants were free to choose templates from any population synthesis models or any SFH). For TESTs 1, 2B and 3B, all the methods used $\mathrm{BC} 03$ evolutionary synthesis models and exponentially declining SFHs. Details are also listed in Table 2. Where possible, we use the same identification for the teams as in Dahlen et al. (2013).

Acquaviva (1.A)—GalMC code (Acquaviva et al. 2011): The algorithm is a Markov Chain Monte Carlo (MCMC) sampler based on Bayesian statistics. In this approach, the SED fitting parameters (age, mass, reddening, and e-folding time for $\tau$ models) are treated as random variables. The parameter space is explored with a random walk biased so that the frequency of visited locations is proportional to the posterior Probability 
Table 3

Details of the Methods and Parameters Used for Stellar Mass Measurement

\section{Method 1.A}

Team ID: 1

PI: Acquaviva

Code ID: A

Code: GalMC (Acquaviva et al. 2011)

Fitting Method: MCMC

Stellar Population Synthesis Templates: CB07 or BC03 (see the text)

Star Formation History: Constant

Extinction law: Calzetti, $E(B-V)=0.0-1.0$

Ages: $10^{6}-1.4 \times 10^{10}$ years

Nebular emission: yes

Metallicity: $Z_{\odot}$ and $0.2 Z_{\odot}$

Method 4.B

Team ID: 4

PI: Finkelstein

Code ID: B

Code: own code

Fitting Method: $\chi^{2}$

Stellar Population Synthesis Templates: CB07

Star Formation History: Exponentially declining, rising $(\tau=0.0001,0.01,0.1$, $1.0,100.0,-0.3,-1.0,-10.0)$ Gyrs

(the negative values correspond to a rising SFR)

Extinction law: Calzetti, $E(B-V)=0.0-0.51$

Ages: $1 \mathrm{Myr}-13$ Gyrs

Nebular emission: yes

Metallicity: $Z_{\odot}$ and $0.2 Z_{\odot}$

Method 6.C

Team ID: 6

PI: Fontana

Code ID: C

Code: own code

Fitting Method: $\chi^{2}$

Stellar Population Synthesis Templates: BC03

Star Formation History: Exponentially declining with $\tau=0.1,0.3,0.6,1,2,3$, 5, 9, 15 Gyrs

Extinction law: Calzetti+SMC, $E(B-V)=0.0-1.1$ in increments of 0.05

Ages: $\log ($ age $)=7-7.35$ (in 0.05 steps), 7.4-8.9 (0.1 steps), 9-10.3 (0.05 steps)

Nebular emission: no

Metallicity: $0.2 Z_{\odot}, 0.4 Z_{\odot}, Z_{\odot}, 2.5 Z_{\odot}$ also a subset of models with age $<1$ Gyrs and $Z_{\odot}=0.02$

Method 7.D

Team ID: 7

PI: Gruetzbauch

Code ID: D

Code: EAZY (Brammer et al. 2008)

Fitting Method: $\chi^{2}$

Stellar Population Synthesis Templates: BC03

Star Formation History: Exponentially declining with $\tau=0.01,0.03,0.06,0.1$, $0.25,0.5,0.75,1.0,1.3,1.7,2.2,2.7,3.25,3.75,4.25,4.75,5.25,5.75,6.25$, $6.75,7.25,7.75,8.25,8.75,9.25,9.75,10.25,10.75$ Gyrs

Extinction law: Calzetti, $A_{v}=0 ., 0.2,0.4,0.6,0.8,1.0,1.33,1.66,2,2.5$

Ages: $1 \mathrm{Myr}-13 \mathrm{Gyrs}$ - ages required to be smaller than the age of the universe at each redshift

Nebular emission: no

Metallicity: $(X, Y, Z):(0.7696,0.2303,0.0001),(0.7686,0.231,0.0004)$, (0.756, 0.24, 0.004), (0.742, 0.25, 0.008), (0.70, 0.28, 0.02), (0.5980,

$0.352,0.0500),(0.4250,0.475,0.1000)$

Method 8.E

Team ID: 8

PI: Johnson

Code ID: E

Code: SATMC (Johnson 2013)

Fitting Method: MCMC
Table 3

(Continued)

Stellar Population Synthesis Templates: BC03

Star Formation History: Instantaneous burst

Extinction law: Calzetti, $E(B-V)=0.0-4.5$

Ages: $0.01-10 \mathrm{Gyr}$ (unequally spaced and taken directly from BC03 library)

Nebular emission: no

Metallicity: $0.0001 Z_{\odot}, 0.0004 Z_{\odot}, 0.004 Z_{\odot}, 0.02 Z_{\odot} 0.05 Z_{\odot}$

Method 9.F

Team ID: 9

PI: Papovich

Code ID: $\mathrm{F}$

Code: Own Code

Fitting Method: $\chi^{2}$

Stellar Population Synthesis Templates: BC03

Star Formation History: Exponentially declining $(\tau=0.001,0.01,0.03,0.1$, $0.3,1.0,3.0,10.0,100.0 \mathrm{Gyr})$

Extinction law: Calzetti

Ages: 0.0251, 0.04, 0.064, 0.1015, 0.161, 0.255, 0.6405, 1.0152, 1.609, 2.5, 4.0, 6.25 and $10.0 \mathrm{Gyrs}$

Nebular emission: no

Metallicity: Solar, except for TEST-2A for which the following are used: 0.02, $0.2,0.4,1.0,2.5 Z_{\odot}$

Method 10.G

Team ID: 10

PI: Pforr

Code ID: G

Code: HyperZ (Bolzonella et al. 2000)

Fitting Method: $\chi^{2}$

Stellar Population Synthesis Templates: M05

Star Formation History: Exponentially declining $(\tau=0.1,0.3,1.0 \mathrm{Gyr})$,

Constant SF at $t=0.1,0.3,1 \mathrm{Gyr}$,

zero SF afterwards, Constant star formation

Extinction law: none

Ages: 0-20 Gyr (221 in total, grid as in BC03 templates)

Nebular emission: no

Metallicity: $0.2 Z_{\odot}, 0.5 Z_{\odot}, 1.0 Z_{\odot}, 2 Z_{\odot}$

Method 11.H

Team ID: 11

PI: Salvato

Code ID: $\mathrm{H}$

Code: Le Phare (Arnouts \& Ilbert 2011)

Fitting Method: $\chi^{2}$

Stellar Population Synthesis Templates: BC03

Star Formation History: Exponentially declining $(\tau=0.1,0.3,1,2,3,5,10,15$, $30 \mathrm{Gyr})$

Extinction law: Calzetti

Ages: 0.01-13.5 Gyr

Nebular emission: yes

Metallicity: $0.02 Z_{\odot}, 0.008 Z_{\odot}$

Method 12.I

Team ID: 12

PI: Wiklind

Code ID: I

Code: Own Code (Wiklind et al. 2008)

Fitting Method: $\chi^{2}$

Stellar Population Synthesis Templates: BC03

Star Formation History: Exponentially declining $(\tau=0.1,0.2,0.3,0.4,0.6,0.8$, $1.0 \mathrm{Gyr})$ and instantaneous burst $(\tau=0)$

Extinction law: Calzetti

Ages: $0.05,0.1,0.2,0.3,0.4,0.5,0.6,0.7,0.8,1.0,1.2,1.4,1.6,1.8,2.0,2.5$, 3.0, 3.5, 4.0, 5.0, 6.0, 7.0 Gyrs

Nebular emission: no

Metallicity: $0.2 Z_{\odot}, 0.4 Z_{\odot}, 1.0 Z_{\odot}, 2.5 Z_{\odot}$

Method 13.J

Team ID: 13 
Table 3

(Continued)

PI: Wuyts

Code ID: J

Code: FAST (Kriek et al. 2009)

Fitting Method: $\chi^{2}$

Stellar Population Synthesis Templates: BC03

Star Formation History: Exponentially declining $(\log (\tau)=8.5-10$ in increments of 0.1

Extinction law: Calzetti

Ages: $\log ($ age $)=7.7$ to 10.1 in increments of 0.1

Nebular emission: no

Metallicity: solar

Density Function (PDF). The desired intervals of the SED fitting parameters are then obtained by marginalizing the PDF, which in MCMC simply corresponds to summing over the points in the chains. Here, a new version of the GalMC is used (SpeedyMC; Acquaviva et al. 2012), which is 20,000 times faster and at every step of the chain, the spectra are generated through multi-linear interpolation of a library of pre-computed models. The best-fit stellar masses and the $68 \%$ uncertainties are predicted from these marginalized distributions.

For TESTs 2A, 3A and 4, this code used templates based on S. Charlot \& G. Bruzual (2007, private communication, hereafter $\mathrm{CB} 07$ ) while for other tests it used BC03 population synthesis models. Two metallicities are used: Solar and $0.2 Z_{\odot}$ with the one giving the optimum $\chi^{2}$ value chosen.

Finkelstein (4.B)—own code: This uses $\chi^{2}$ fitting method with $\mathrm{CB} 07$ population synthesis model. It uses a hybrid SFH (exponentially declining + rising star formation rate).

Fontana (6.C)—own code: This uses $\chi^{2}$ fitting method with the SED templates generated from $\mathrm{BC} 03$ and exponentially declining SFR. The templates are generated with both Calzetti and SMC dust models and hence, the code can choose between the two dust extinction scenarios, whichever gives a better fit.

Gruetzbauch (7.D)_EAZY code-Brammer et al. (2008): Uses $\chi^{2}$ fitting method with $\mathrm{BC} 03$ and an exponentially declining SFR with a large set of $\tau$ values. Also uses a large set of metallicity and extinction values.

Johnson (8.E)—SATMC code-Johnson (2013): Uses the MCMC to fit the SEDs, similar to method 1.A. BC03 templates are used with instantaneous burst of star formation. This is the only experiment which uses this SFH. For the fit, all the parameters in the code are varied.

Papovich (9.F) —own code: this is a $\chi^{2}$ minimization code. It uses an exponentially declining SFR. Solar metallicity is assumed. The code uses templates based on BC03 models.

Pforr (10.G)_HYPERZ code-Bolzonella et al. (2000): this is a $\chi^{2}$ minimization code. It uses hybrid SFH consisting of exponentially declining, truncated and constant SFRs. In this respect, 10.G is different from many of the methods listed in Table 3 but is similar to others (e.g., 4.B). This is the only method which uses Maraston (2005) population synthesis code to generate templates.

Salvato (11.H)_La Phare code-Arnouts \& Ilbert (2011): this uses a $\chi^{2}$ minimization technique and $\mathrm{BCO} 3$ code to generate templates. Exponentially declining star formation rate is used. The prior $E(B-V)<0.15$ is applied if the ratio $t / \tau>4$ (i.e., significant extinction is only allowed for galaxies with high SFR).

Wiklind (12.I) —own code-Wiklind et al. (2008): uses $\chi^{2}$ minimization of the SEDs. The errors in stellar mass are estimated from Monte Carlo simulations. Exponentially declining star formation rates are used with $\tau=0$ representing an instantaneous burst. The template SEDs are based on BC03.

Wuyts (13.J)_FAST code-Kriek et al. (2009): uses $\chi^{2}$ fitting technique with exponentially declining SFR. The templates are from $\mathrm{BC} 03$ with solar metallicity.

Details of individual methods are listed in Table 3. In the next section we compare the input mass with the stellar mass estimates independently measured from different methods (Tables 2 and 3) to explore differences as a function of the method (TEST-1), free parameters (extinction, SFH, age)(TEST-2), templates used and internal consistency (TEST-3) and the photometric depth and selection wavelength (TEST-4). This allows a study of the absolute consistency (i.e., how well each code produces the expected mass) and relative consistency (how the estimated masses between different codes agree). In the following sections, we perform a step-by-step study of the above, using the information in Tables 1 and 3.

\section{TEST-1: COMPARISON OF STELLAR MASSES FROM DIFFERENT METHODS}

\subsection{Dependence on the SED Fitting Codes}

The participating teams, listed in Table 3, used the mock catalog generated for TEST-1 and independently estimated the stellar mass for individual galaxies. For each code, Figure 1 shows changes between the input mass, $\log \left(M_{\text {input }}\right)$, and $\Delta(\log M)$, defined as the difference between the input mass and stellar mass estimated from that code, $M_{\text {est }}$ : $\Delta(\log M)=\log \left(M_{\text {input }}\right)-\log \left(M_{\text {est }}\right)$. The very small scatter in the case of 1.A is to be expected because TEST-1 mock catalog was generated based on this method and therefore it confirms the consistency between the input and estimated masses. As a result, the observed scatter in the stellar mass from method 1.A is likely due to the effect of photometric errors added to the mock data. This is supported by the results from Figure 2(a) which shows an increase in the scatter in $\Delta \log (M)$ based on method 1.A from bright to faint magnitudes (see below). Figure 1 also confirms that all the methods used in this experiment recover the input mass values to good accuracy. There are no systematic effects or mass-dependent biases, indicating that none of the methods in Table 3 is significantly biased.

It is clear from Figure 1 that for most of the methods, the scatter reduces toward the higher mass end $\left(M>10^{10} M_{\odot}\right)$. This is because these galaxies are often brighter with a higher photometric S/N. This is demonstrated in Figure 2(a), where we study changes in $\Delta \log (M)$ as a function of $H$-band $(F 160 W)$ magnitude, showing an increase in the scatter at $H_{\mathrm{AB}}>26 \mathrm{mag}$. This indicates that the main source of inconsistency between the stellar mass estimates among different codes, keeping everything else the same, is for the relatively fainter galaxies (and those with lower photometric S/ $\mathrm{N})$ and due to different ways the photometric errors are handled in the SED fitting process. Figure 2(b) shows the change in $\Delta \log (M)$ as a function of redshift where, for most of the methods, we find no correlation and hence, no redshiftdependent biases. The exception is 7.D where shows a bias at 


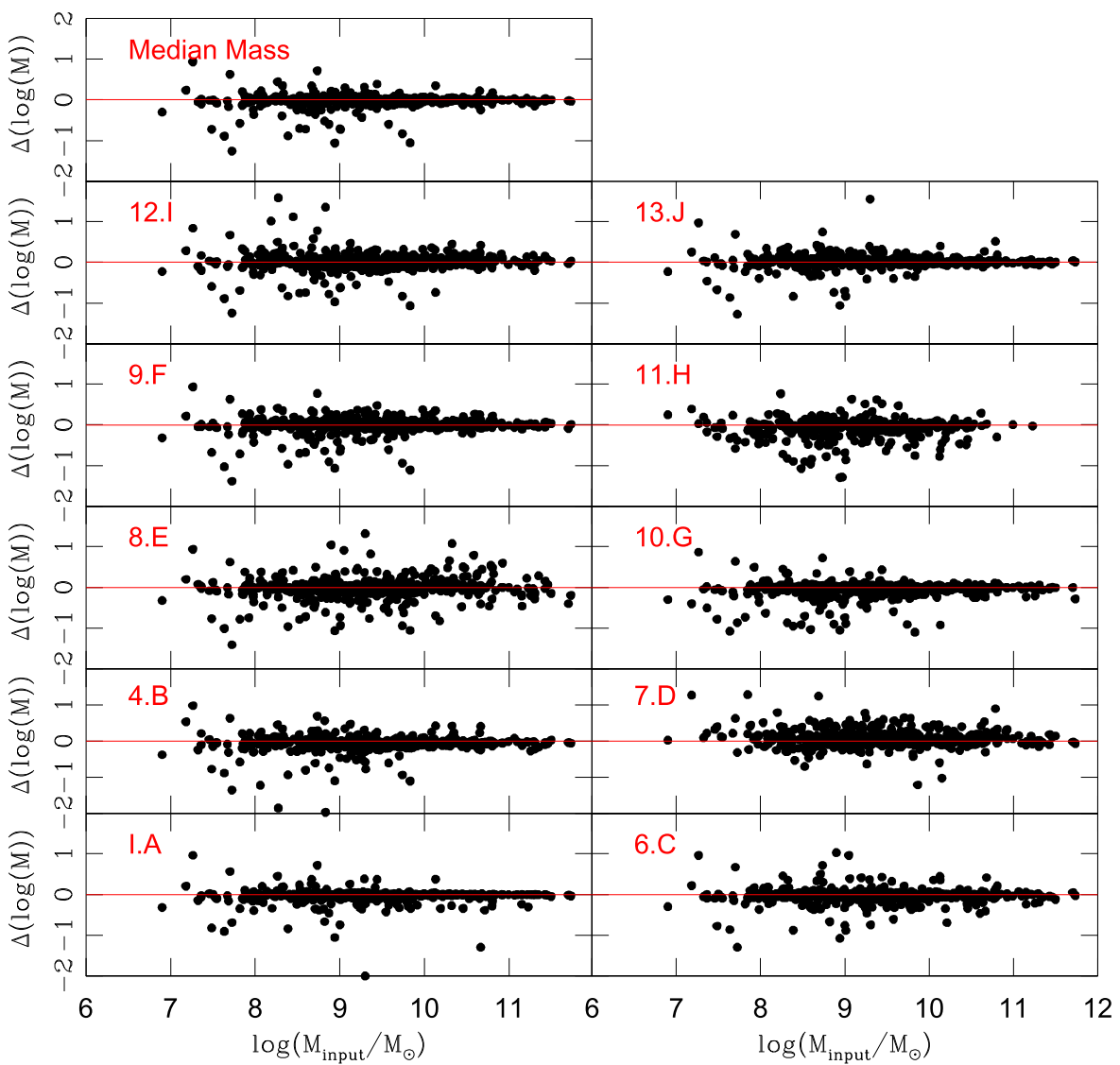

Figure 1. Stellar mass difference $(\Delta \log (M))$ as a function of $\log \left(M_{\text {input }}\right)$ measured from all the participating methods, using TEST-1. $\Delta \log (M)$ is defined as $\Delta \log (M)=\log \left(M_{\text {input }}\right)-\log \left(M_{\text {est }}\right)$ where $M_{\text {est }}$ is the estimated stellar mass. The red horizontal line shows the expected relation if the input stellar mass is exactly produced. A total of 559 simulated galaxies are used. This test examines the sensitivity of the stellar mass to the methods/codes listed in Table 3.

$z<1$. The reason for the observed redshift-dependent bias here is not clear but is likely due to degeneracy caused by using a wide range of metallicities. The redshift distribution in TEST-1 catalog, presented in Appendix B, is fixed to be the same as the observed (spectroscopic) distribution for the GOODS-S field. Therefore, the results from this study are directly applicable to the observed samples.

For each method, we estimated the rms, outlier fraction and the bias in $\Delta \log (M)$ values using galaxies in the mock catalogs (i.e the scatter in $\Delta \log (M)$ from individual methods among all the galaxies). Results are listed in Table 4. The outlier fraction is defined as the ratio of the number of galaxies with $|\Delta \log (M)|>0.5$ to the total number of galaxies while the bias factor is defined as mean $[\Delta \log (M)]$. Overall, there is good agreement between the estimated masses from different methods and the input mass. The rms values range from $0.141 \mathrm{dex}(13 . \mathrm{J})$ to $0.241 \mathrm{dex}(11 . \mathrm{H})$. The highest rms values and outlier fractions are for methods 7.D, 8.E and 11.H. Method 8.E uses the MCMC technique, which is different from what used in other methods (except for method 1.A). Both 7.D and $11 . \mathrm{H}$ also show higher biases $(-0.059$ and 0.057 respectively), contributing to the relatively higher rms scatter. All these codes have relatively low resolution $E(B-V)$ and age grids. The lowest rms scatter is associated with codes: 6.C, 10.G and 13.G which have a relatively higher resolution in $E(B-V)$ and age grids.

Figure 3 shows changes in the rms, outlier fraction and bias as a function of the $\mathrm{S} / \mathrm{N}$. There is a clear reduction in the rms and outlier fractions with increasing $\mathrm{S} / \mathrm{N}$. However, the estimated bias from all the methods is found to be independent of the $\mathrm{S} / \mathrm{N}$, with significant reduction in the bias when the outliers are removed. This supports our earlier conclusion that some of the differences in the stellar mass measurements from different methods could be attributed to low $\mathrm{S} / \mathrm{N}$ in the photometric data.

We note that there is good agreement between the input and estimated stellar mass values when using the median of stellar masses measured for individual galaxies from different methods $\left(M_{\text {med }}\right)$. The rms in $\Delta \log \left(M_{\text {med }}\right)$ is 0.142 dex where $\Delta \log \left(M_{\text {med }}\right)=\log \left(M_{\text {input }}\right)-\log \left(M_{\text {med }}\right)$. However, the median will be affected if some of the methods are biased. As shown in Table 4 and Figures 2(a) and (b), for most of the methods, there is no indication of significant bias in the masses. Since the same input parameters are used for all the experiments in TEST-1, the median of the mass estimates for each galaxy measured from different methods is less affected by code-dependent uncertainties. Therefore, the smaller rms for the median suggests that the numerical noise (presumably due to different approximations and interpolations made in the fitting codes) is reduced by combining results from different methods. This numerical noise is small compared to other systematic uncertainties, so the gain from taking the median rather than using a single, well tested, code is likely to be useful only when values based on the same underlying set of assumptions are desired.

The rms scatter measured for the stellar masses in TEST1 are based on galaxy samples which cover a range in luminosities and photometric $\mathrm{S} / \mathrm{N}$ and also methods which 

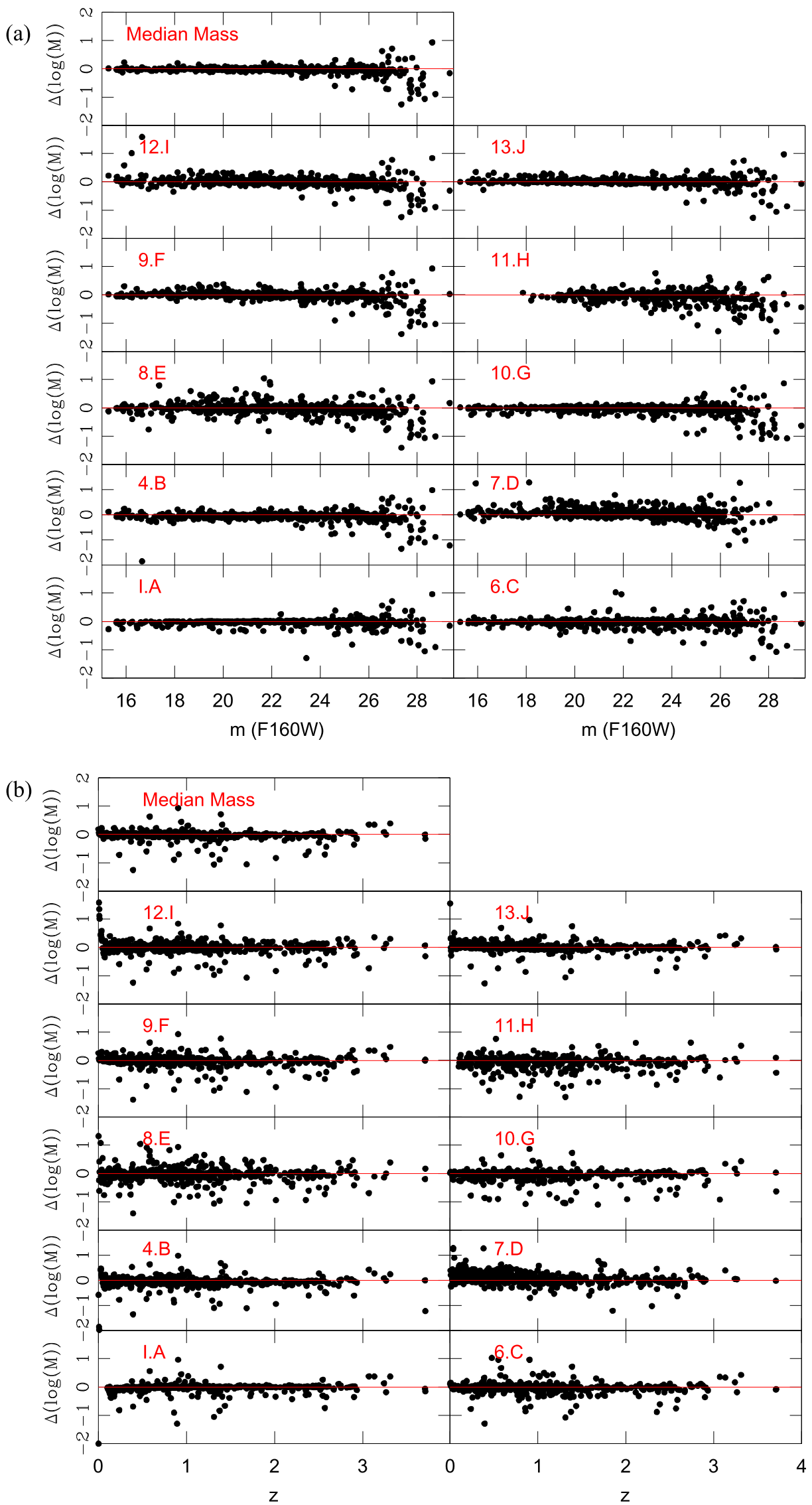

Figure 2. 2(a) Top panel: dependence of $\Delta \log (M)$ on $H$-band (F160W) magnitudes in TEST-1, showing sensitivity of $\Delta \log (M)$ on the photometric S/N. 2(b) Bottom panel: the same as 2(a) but for redshift. All the input parameters in this test are fixed with the only variable being the codes/methods. These show the sensitivity of the stellar mass on the codes over the range of magnitudes and redshifts covered. 
Table 4

The rms Scatter, Bias and Outlier Fraction (OLF) in $\Delta \log (M)$ for Mock Sample in TEST-1

\begin{tabular}{lccrc}
\hline \hline Code & rms & $\begin{array}{c}\text { rms } \\
\text { No Outliers }\end{array}$ & Bias $^{\mathrm{a}}$ & $\begin{array}{c}\text { Outlier }^{\mathrm{a}} \\
\text { Fraction }\end{array}$ \\
\hline 1.A & 0.134 & 0.100 & 0.026 & 0.014 \\
4.B & 0.175 & 0.127 & 0.047 & 0.030 \\
6.C & 0.167 & 0.124 & 0.023 & 0.021 \\
7.D & 0.214 & 0.177 & -0.059 & 0.040 \\
8.E & 0.228 & 0.164 & 0.010 & 0.049 \\
9.F & 0.180 & 0.110 & 0.024 & 0.034 \\
10.G & 0.172 & 0.123 & 0.000 & 0.026 \\
11.H & 0.241 & 0.172 & 0.057 & 0.060 \\
12.I & 0.181 & 0.129 & -0.014 & 0.032 \\
13.J & 0.141 & 0.106 & -0.018 & 0.015 \\
Median & 0.142 & 0.089 & 0.010 & 0.024 \\
\hline
\end{tabular}

Note.

${ }^{\text {a }}$ Outlier fraction is defined as the ratio of the number of galaxies with $\Delta(\log (M))>0.5$ to the total number of galaxies where $\Delta \log (M)=$ $\log \left(M_{\text {input }}\right)-\log \left(M_{\text {est }}\right)$. The bias is defined as mean $[\Delta \log (M)]$.

handle these errors differently. This also contributes to the rms values in Table 4. To quantify this, we measure the rms in $\Delta \log (M)$ for individual galaxies in the mock catalog from each method separately. In this case, the rms in $\log \Delta(M)$, estimated for each galaxy from different methods, represents the genuine scatter among different codes/methods, only depending on the way each code/method treats the photometric error. Changes in the rms scatter as a function of the S/N for galaxies in TEST-1 is presented in Figure 4. Given that for a single galaxy the photometric errors are fixed, the relation between the rms values in $\Delta \log (M)$ (corresponding to individual galaxies as measured from different codes) and the $\mathrm{S} / \mathrm{N}$ reveals the extent to which handling the photometric errors by each code affects the resulting stellar mass estimates. The rms reduces with increasing the $\mathrm{S} / \mathrm{N}$ and asymptotes around $\mathrm{rms}=0.05 \mathrm{dex}$ (at $\mathrm{S} / \mathrm{N}>40$ ), where the photometric uncertainties become very small. This gives a measure of the systematic effects in the stellar mass measurement entirely due to the methods used (when all the rest of the parameters are fixed and photometric errors are negligible).

The rms in $\Delta \log (M)$, estimated for method 1.A (Table 4), is mainly due to contribution from photometric errors in stellar mass measurement and not the method or the SED templates used (because the template SEDs in TEST-1 were generated by this code and were used again to estimate the observable parameters after introducing photometric noise to the SEDs). Therefore, we estimate the intrinsic uncertainty in $\Delta \log (M)$ associated with each method (in Table 4), $\sigma_{\text {method, } i \text {, as }}$ $\sqrt{\sigma_{i}^{2}-\sigma_{1 . \mathrm{A}}^{2}}$ where, $\sigma_{i}$ and $\sigma_{1 . \mathrm{A}}$ are the rms values for individual methods and for method 1.A (corresponding to photometric uncertainties) respectively. Here we assume that differences in $\sigma_{i}$ due to treatment of age and extinction among different codes is negligible (however, see the next section). The total uncertainty in $\Delta \log (M)$ due to differences in codes/methods used is therefore, $\sigma_{m}=\frac{1}{\sqrt{n}} \sqrt{\sum_{i=1}^{n} \sigma_{\text {method }, i}^{2}}=0.136$ dex, where $n$ is the number of methods/codes used. Using the median rms value from all the methods (Table 4), we estimate $\sqrt{\sigma_{\text {median }}^{2}-\sigma_{1 . \mathrm{A}}^{2}}=0.047 \mathrm{dex}$. This is close to $\mathrm{rms}=0.050 \mathrm{dex}$ we estimated for systematic errors from Figure 4 and is significantly smaller than the rms scatter of 0.136 dex due to different methods/codes used. This confirms that the median mass (among all the methods/codes) provides the closest estimate to the "real" stellar mass.

A Spearman Ranking Test was performed between the input, $M_{\text {input }}$, and estimated mass, $M_{\text {est }}$, for each galaxy as measured by applying different codes on the mock sample in TEST-1. Combined with the Pearson correlation coefficients from this test, as listed in Table 5, this confirms very close ranking of the stellar masses measured from different codes (i.e., the codes consistently produce the mass sequence for galaxies in the catalog).

We conclude that the uncertainties in the estimated stellar mass are dependent on the resolution of color excess $\left(E_{B-V}\right)$ and age grids as well as the photometric S/N. We find an rms scatter of 0.135 dex in $\Delta \log (M)$ due to code-dependent effects. The estimated uncertainty in $\log (M)$ due to photometric errors is 0.134 dex while using the median mass, it reduces to 0.05 dex. No evidence is found for bias in any of the methods in Table 4. For each galaxy, the median stellar mass between different methods gives the most accurate stellar mass with the errors mainly dominated by systematic effects.

\subsection{TEST-1: The Effect of Age and Extinction on Stellar Mass Estimates}

A serious problem in stellar mass measurement for galaxies through SED fitting is the interplay between the mass, age and extinction, leading to correlated errors among these parameters. The problem is compounded by the fact that there is no direct and model-independent measure for these parameters, although there is independent constraint on extinction with mid to far-IR dust measurements (e.g., Reddy et al. 2012), which narrows the range of allowed age and extinction values. Therefore, the only way to constrain them is through simulations, where we know a priori the input values for each galaxy. The mock catalog in TEST-1 also provides the input age and extinction for each simulated galaxy, providing a reference with which to compare their predicted values. In this section we study the uncertainty introduced to the estimated stellar mass values due to the interplay between age and extinction. Here age is defined as the time since the on-set of star formation and an exponentially declining SFH is assumed.

Figure 5 shows the dependence of $\Delta \log (M)$ on the input (expected) age and extinction $\left(E_{(B-V)}\right)$ for different methods. The sample is divided into three different age and $E_{B-V}$ bins (corresponding to their input values from the simulation). On the $\Delta \log (M)-E_{B-V}$ and $\Delta \log (M)-\log ($ age $)$ plots, these respectively correspond to: $7<\log$ (age) $<8$ (blue); $8<\log$ (age) $<9$ (black); $9<\log ($ age $)<10$ (red) and $0<E_{B-V}$ $<0.3$ (blue); $0.3<E_{B-V}<0.6$ (black); $E_{B-V}>0.6$ (red). There is significant scatter in $\Delta \log (M)$ at a given age or extinction interval. As expected, the number of old galaxies (age $>10^{9}$ years) with high extinction is small. In particular, the scatter is higher for younger galaxies, independent of the extinction.

For some of the models (6.C, 8.E, 10.G, 11.H, 12.I) in Figure 5, we find a sequence of galaxies with ages $<10^{8}$ ) years clearly separated from the $\Delta \log (M)=0$ line. These galaxies all have wrong stellar masses (i.e., large $\Delta \log (M)$ values). Furthermore, this does not depend on a particular code and SED fitting method as many of the methods show the same 

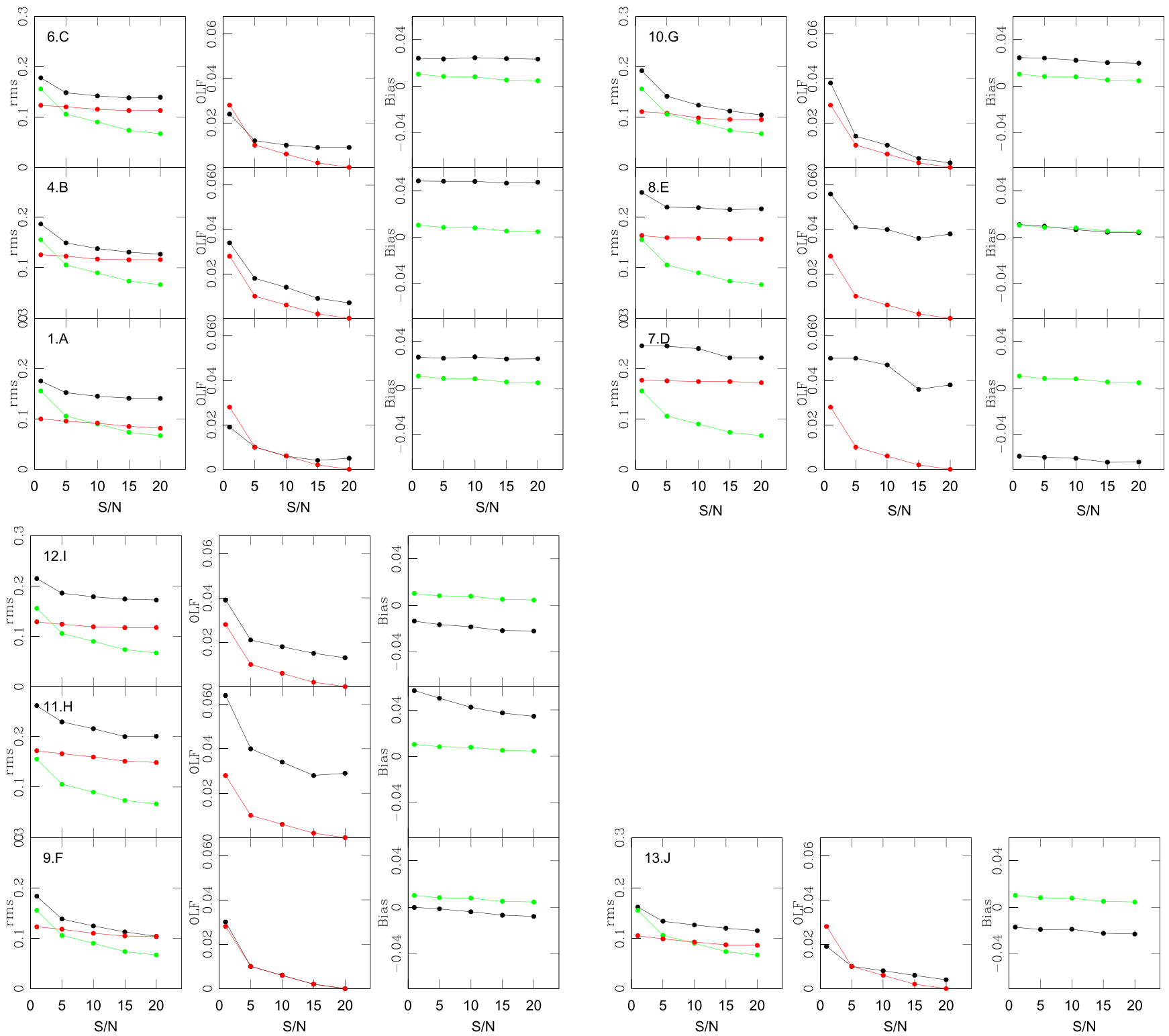

Figure 3. Shows changes in the rms (left), outlier fractions (middle) and bias (right) for different methods in TEST-1, as a function of the photometric S/N. Different colors represent estimates for the whole sample (black line), with the outliers removed (green line) and those corresponding to the median mass (red lines). The $\mathrm{S} / \mathrm{N}$ is measured from the $F 160 \mathrm{~W}$ band.

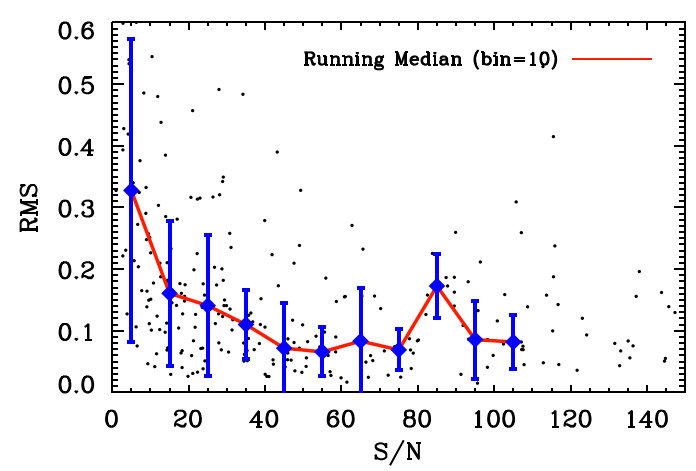

Figure 4. Changes in the rms with $\mathrm{S} / \mathrm{N}$ values. The rms is the scatter in stellar mass values $(\Delta \log (M))$ for individual galaxies in TEST-1, based on different methods. The filled circles are the median values in $\mathrm{S} / \mathrm{N}$ bins with the errorbars corresponding to Poisson statistics. The scatter at a given $\mathrm{S} / \mathrm{N}$ represents the dispersion in the stellar mass values among different methods.
Table 5

Estimated Spearman Rank Coefficients (Column 1) and Pearson Correlation Coefficients (Column 2) for TEST-1, Using $M_{\text {input }} / M_{\odot}$ as the Reference Mass

\begin{tabular}{lcc}
\hline \hline Code ID & 1 & 2 \\
\hline 1.A & 0.91 & 0.92 \\
4.B & 0.98 & 0.96 \\
6.C & 0.98 & 0.98 \\
7.D & 0.96 & 0.82 \\
8.E & 0.96 & 0.96 \\
9.F & 0.98 & 0.98 \\
10.G & 0.98 & 0.98 \\
11.H & 0.96 & 0.95 \\
12.I & 0.96 & 0.96 \\
13.J & 0.99 & 0.98 \\
\hline
\end{tabular}

Note. (1). Spearman rank correlation coefficient (2). Pearson correlation coefficient. 

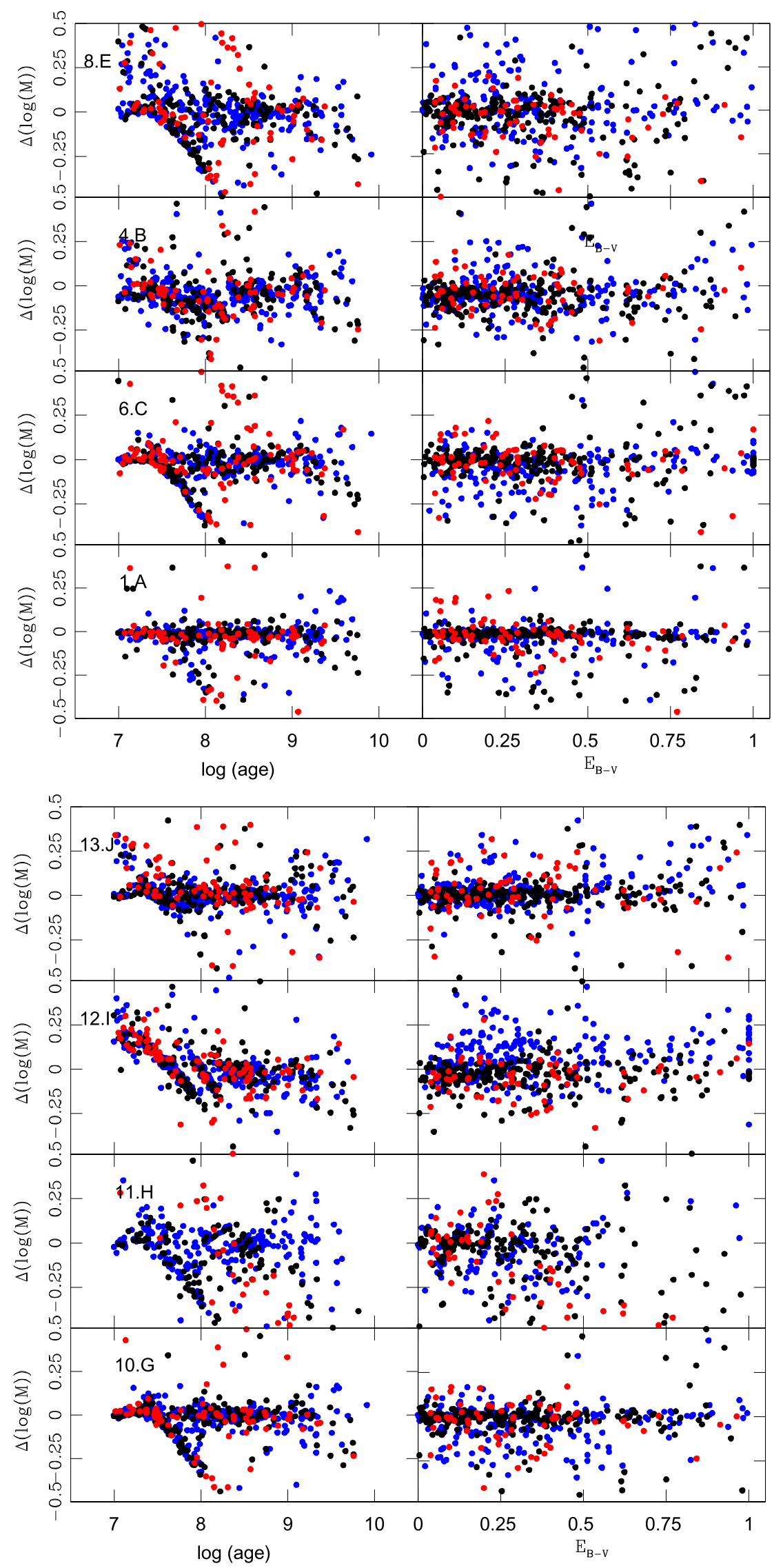

Figure 5. Deviations in the stellar mass estimates from their input values $\left(\Delta \log (M)=\log \left(M_{\text {input }}\right)-\log \left(M_{\text {est }}\right)\right)$ from TEST-1 are plotted against the input age and extinction. Left panels: objects are divided into three different extinction intervals $-0<E_{B-V}<0.3$ (blue), $0.3<E_{B-V}<0.6$ (black); $E_{B-V}>0.6$ (red). Right panels: objects are divided into three different age intervals $-7<\log ($ age $)<8$ (blue) $; 8<\log ($ age) $<9$ (black); $9<\log ($ age $)<10$ (red). This separates the contributions due to age, extinction and code/method to errors in the stellar mass estimates. 
sequence. To find about sources of uncertainty in the stellar mass measurement, we need to understand the cause of such deviations. A large fraction of the deviant galaxies have intermediate to high extinctions $(E(B-V)>0.3)$ indicating they are likely dusty starburst systems. The degeneracy between the SED fitting parameters for these galaxies is higher as their SEDs mimic both the dusty starbursts and quiescent systems.

We now explore the extent to which age and extinction are responsible for the sequences seen in Figure 5 and for uncertainties in stellar mass measurement. Using the input age and extinction values for simulated galaxies in TEST-1, we compare $\Delta \log (M)$ with both $\Delta \log ($ age $)$ and $\Delta\left(E_{B-V}\right)$ (respectively defined as $\Delta \log ($ age $)=\log \left(\right.$ age $\left._{\text {est }}\right)-\log$ (age $\left._{\text {input }}\right)$ and $\left.\Delta\left(E_{B-V}\right)=E_{(B-V), \text { est }}-E_{(B-V) \text {,input }}\right)$ for each method, with results presented in Figure 6. All the experiments show a strong correlation between deviations in the stellar mass and age. This indicates that galaxies with uncertain stellar mass estimates also have uncertain ages (i.e., large $\Delta \log (M)$ and $\Delta$ (age) values). In other words, the errors in the stellar mass and age for mock galaxies, when constraining other parameters (as in TEST-1), are correlated. The observed divergence between the age estimates for younger galaxies $\left(<10^{8}\right.$ years $)$ is partly due to the varying $M / L$ among these systems. The observed trend in Figure 6 is somewhat weaker on the $\Delta \log$ $(M)$ versus $\Delta E(B-V)$ plane, indicating that for the range in $-0.5<\Delta E(B-V)<0.5$, there is a wide range in $\Delta \log$ $(M)-(-1<\Delta \log (M)<1)$, caused by differences in the estimated age values. By constraining galaxies only to those with ages $>10^{8}$ years, the observed trend in Figure 6 in both extinction and stellar mass is reduced.

Given the degeneracy between age and extinction and to understand the error budget in the stellar mass estimates, we now disentangle contributions from these parameters by dividing the sample into three different age and extinction intervals and estimating the rms in $\Delta \log (M)$ values for each interval. The result is a covariance matrix representing the error budget where the rows and columns are the age and extinction respectively, with the matrix elements being the rms values in $\Delta \log (M)$ i.e., the stellar mass within a given age-extinction grid. As in Figure 5, the sample is divided into age and extinction intervals: $10^{7}<$ age $<10^{8} ; 10^{8}<$ age $<10^{9}$; $10^{9}<$ age $<10^{10}$ years and $E_{B-V}<0.3 ; 0.3<E_{B-V}<0.6$; $E_{B-V}>0.6$. The error budget matrices corresponding to each of the methods are presented in Table 6. For any given method, the elements of the matrix correspond to the rms scatter in $\Delta \log (M)$ for a given age and extinction. Using these error budget matrices, we separate relative contributions due to method, age and extinction to observed uncertainties in the stellar mass.

Overall, the methods agree well per age-extinction grid. Also, for any given method, the rms scatter in $\Delta \log (M)$ (Table 6) increases for redder $(E(B-V)>0.6)$ and older (age $>10^{9}$ years) galaxies. The total error budget matrix (the overall uncertainty in $\Delta \log (M)$ for different age and $E(B-V))$ from all methods combined, is estimated as $\sigma_{i j}=\frac{1}{\sqrt{n}} \sqrt{\sum_{k=1}^{k=n} \sigma_{i j, k}^{2}}$, where $\sigma_{i j, k}$ are the matrix elements at any given age, $i$, and extinction, $j$, grid corresponding to the method, $k$. The total error budget matrix is also given in Table 6.
The rms scatter in $\Delta \log (M)$ from method 1.A is likely dominated by photometric errors. Therefore, the error matrix associated with this method in Table 6 provides a lower limit to uncertainties in the stellar mass measurement (for any age/ extinction combination) caused by photometric errors.

In conclusion, we find that uncertainties in stellar mass measurement are coupled with those in age and extinction, being more tightly coupled with errors in age. The same galaxies are outliers in both stellar mass and age regardless of the code used. We find serious degeneracy for galaxies with ages $<10^{8}$ years, with the rms scatter in stellar mass increasing for redder and older systems. Relative contributions due to age and extinction are disentangled by forming a covariance matrix.

\section{TEST-2: EFFECT OF FREE PARAMETERS ON STELLAR MASS MEASUREMENT}

The tests performed in the last section were used to quantify the deviation in the estimated mass of galaxies (from their expected values) due to different methods and to disentangle the effects of age and extinction in stellar mass measurement. Here, we explore the effect of free parameters (i.e., degeneracies in the SED fits) on the stellar mass estimates. First, we perform SED fits to the mock data, allowing all the parameters to be free (except for the IMF which is chosen to be Chabrier and the redshift, which is fixed to its input value)(TEST-2A). Second, we fix all the parameters in the SED fits and repeat the analysis (TEST-2B). The participating teams estimated the stellar masses following the above prescriptions. By comparing results between TEST2-A and TEST-2B for each method, we eliminate the code-dependent effects. The difference then reveals the effect of free parameters on the stellar mass estimate.

Figures 7(a) and (b) compare the input and estimated stellar mass values from different methods for TEST-2A and TEST$2 \mathrm{~B}$ respectively. The rms scatter, bias and outlier fractions are estimated and presented in Table 7. For some of the methods in TEST-2A, there is a clear bi-modality between the expected and estimated stellar mass values (e.g., 1.A, 4.B and 6.C). All the methods underpredict the stellar masses at $M<10^{8} M_{\odot}$, with the rms values changing among the methods from 0.172 to 0.394 dex. Also, some of the methods show a systematic offset in the estimated stellar mass from their "true" values. In Figure 7(a), we also examine the distribution of galaxies as a function of extinction, measured for individual galaxies$E_{(B-V)}=0$ (green); $0<E_{B-V}<0.3$ (blue); $0.3<E_{B-V}<0.6$ (black); $E_{B-V}>0.6$ (red). There are two clear sequence of galaxies on the mass comparison plots in Figure 7(a) (TEST2A), separated depending on their extinction values. The sequence is particularly evident for mrthods 1.A, 4.B, 6.C and 10.G. For 1.A, there is a clear separation of galaxies depending on their extinction, with redder galaxies $\left(E_{B-V}>0.3\right)$ having a smaller (estimated) mass. Similar effects are found for experiments 4.B and 6.C where there is a complete absence of sources with high extinction $\left(E_{B-V}>0.6\right)$. Also, sources with medium extinction $\left(0.3<E_{B-V}<0.6\right)$ are mostly associated with galaxies with higher stellar masses. This indicates a possible interplay between stellar mass and extinction when both parameters are estimated simultaneously through the SED fits.

The observed bi-modality disappears in TEST-2B (Figure 7(b)) when the free parameters are fixed. However, there is a mass-dependent effect in TEST-2B where most of the 


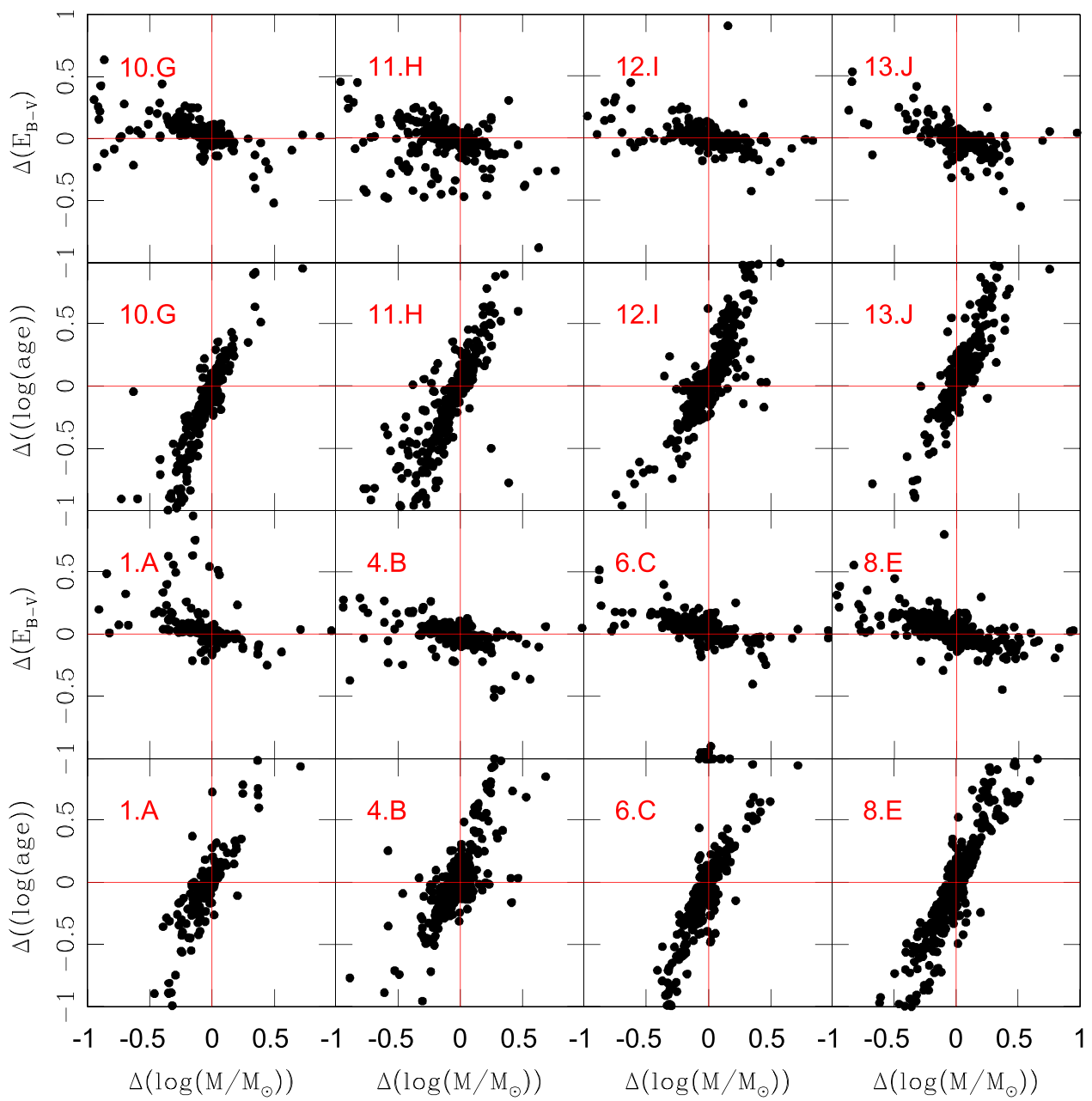

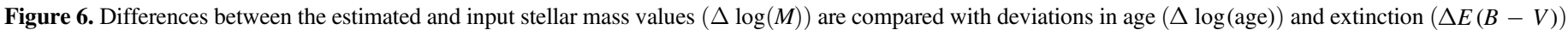

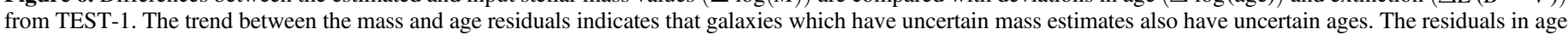
range from -1 to 1 dex while for the extinction they span the range -0.5 to 0.5 dex.

methods underestimate the stellar mass for low $\left(M<10^{8} M_{\odot}\right)$ and high $\left(M>3 \times 10^{9} M_{\odot}\right)$ mass systems. TEST-2B confirms that the observed bi-modality detected in TEST-2A is likely caused by the interplay between the free parameters. The rms in $\Delta \log (M)$ values between the two tests are comparable, with TEST-2B having slightly higher rms (Table 7). Both 1.A and 4.B have higher rms values. They use templates generated from CB07 population synthesis models (for TEST-2A), which is different from the $\mathrm{BC} 03$ model templates used to generate the mock catalog. Furthermore, 1.A and 4.B use constant and hybrid SFHs (for TEST-2A) respectively, which is different from the exponentially declining model assumed for the majority of the methods here. Once the population synthesis model used to generate the template SEDs are adopted consistently with those for the mock data (BC03), as in TEST-2B, the observed bi-modalities disappear (Figure 7(b) also see Section 6.2). However, for almost all the methods there is a relatively higher bias in TEST-2B compared to TEST-2A.

There is a significant offset in the result for the experiment 10.G in TEST-2A, corresponding to a bias of 0.183 dex. This method uses templates generated from Maraston (2005) with a hybrid SFH (consisting of exponentially declining, constant at $0.1,0.3$ and 1 Gyrs and zero afterwards)-(Figure $7(\mathrm{a})$ ). The offset is completely removed in TEST-2B where BC03 was adopted. The observed offset in 10.G shows the sensitivity of the results to template SEDs generated from the two population synthesis codes (BC03 versus M05). The templates resulting from Maraston (2005) include contributions from pulsating Asymptotic Giant Branch (AGB) stars, making them different from the templates based on the $\mathrm{BC} 03$ code, which include less contribution from these stars. This leads to an underestimation of the stellar mass of galaxies when including the AGB contribution in the SEDs. The scatter in method 11.H and 13.J, based on TEST-2A, are small with no offsets observed. These methods both use a SFH and synthetic population models similar to those adopted in TEST-2A. It is clear from Figures $7(\mathrm{a})$ and (b) that using the median of all measured stellar masses, gives smaller rms errors when compared to the expected stellar mass. However, we note that the median stellar mass measured for TEST-2A is not meaningful since the masses from this test are based on different input parameters (i.e., population synthesis models).

For each method, we estimate the difference in quadrature between the rms values for TEST-2A and TEST-2B $\left(\operatorname{rms}[2 \mathrm{~A}-2 \mathrm{~B}]=\sqrt{\sigma_{2 \mathrm{~A}}^{2}-\sigma_{2 \mathrm{~B}}^{2}}\right)$ and present it in Table 7 . This gives the contribution to the error budget in the stellar mass due to degeneracy in the SED fits and changes from 
Table 6

Error Budget Matrices for the Methods in Table 3, when Applied to TEST-1

\begin{tabular}{|c|c|c|c|c|}
\hline Method & $E_{B-V}$ & $<0.3$ & $0.3-0.6$ & $>0.6$ \\
\hline & $\log ($ Age $)$ & & & \\
\hline \multicolumn{5}{|l|}{ 1.A } \\
\hline & 7.5 & 0.074 & 0.100 & 0.251 \\
\hline & 8.5 & 0.076 & 0.169 & 0.250 \\
\hline & 9.5 & 0.167 & 0.267 & 0.165 \\
\hline \multicolumn{5}{|l|}{ 4.B } \\
\hline & 7.5 & 0.112 & 0.155 & 0.242 \\
\hline & 8.5 & 0.128 & 0.168 & 0.310 \\
\hline & 9.5 & 0.232 & 0.242 & 0.394 \\
\hline \multicolumn{5}{|l|}{$6 . \mathrm{C}$} \\
\hline & 7.5 & 0.109 & 0.120 & 0.265 \\
\hline & 8.5 & 0.072 & 0.168 & 0.321 \\
\hline & 9.5 & 0.221 & 0.229 & 0.164 \\
\hline \multicolumn{5}{|l|}{ 8.E } \\
\hline & 7.5 & 0.176 & 0.197 & 0.344 \\
\hline & 8.5 & 0.156 & 0.220 & 0.374 \\
\hline & 9.5 & 0.322 & 0.157 & 0.346 \\
\hline \multicolumn{5}{|l|}{$10 . \mathrm{G}$} \\
\hline & 7.5 & 0.116 & 0.122 & 0.230 \\
\hline & 8.5 & 0.109 & 0.173 & 0.314 \\
\hline & 9.5 & 0.292 & 0.234 & 0.504 \\
\hline \multicolumn{5}{|l|}{ 11.H } \\
\hline & 7.5 & 0.130 & 0.158 & 0.501 \\
\hline & 8.5 & 0.136 & 0.236 & 0.313 \\
\hline & 9.5 & 0.385 & 0.452 & 0.585 \\
\hline \multicolumn{5}{|l|}{ 12.I } \\
\hline & 7.5 & 0.138 & 0.144 & 0.250 \\
\hline & 8.5 & 0.115 & 0.147 & 0.237 \\
\hline & 9.5 & 0.278 & 0.246 & 0.440 \\
\hline \multicolumn{5}{|l|}{ 13.J } \\
\hline & 7.5 & 0.087 & 0.104 & 0.275 \\
\hline & 8.5 & 0.073 & 0.133 & 0.228 \\
\hline & 9.5 & 0.223 & 0.244 & 0.281 \\
\hline \multicolumn{5}{|c|}{ Total Error Budget } \\
\hline & 7.5 & 0.121 & 0.141 & 0.307 \\
\hline & 8.5 & 0.112 & 0.180 & 0.297 \\
\hline & 9.5 & 0.273 & 0.271 & 0.387 \\
\hline
\end{tabular}

Note. The "total" error budget matrix represents the uncertainties for any given age-extinction grid regardless of the code/method. The uncertainties in stellar mass estimates due to photometric errors correspond to the error budget matrix associated with method 1.A.

$0.037 \mathrm{dex}$ (for 6.C) to $0.264 \mathrm{dex}$ (for 9.F). In Figure 8 we compare results between different methods, expressed by their rms and bias (in stellar mass) estimates, as listed in Table 7. The smallest rms value is associated with methods $11 . \mathrm{H}, 12 . \mathrm{I}$ and 13.J as well as the smallest outlier fractions. Method 13.J also has the least bias, indicating that this method provides the closest mass estimates to the "real" values.

The simulations in TEST-2A are the most realistic. Therefore, it is instructive to further investigate the main sources of scatter in $\Delta \log (M)$ values based on this test. In Figure 9 we show $\Delta \log (M)$ distributions as measured from TEST-2A, plotted in $H$-band $(F 160 W)$ magnitude intervals for each method separately. It is clear that for any given method, there is an increase in the width of the distributions from bright to faint magnitudes, indicating the effect of photometric $\mathrm{S} / \mathrm{N}$ on the stellar mass measurement. For some methods, there is an offset from $\Delta \log (M)=0$, likely caused by systematic effects in stellar mass measurement. There are also differences in the distributions among different methods even over the same luminosity range. Figure 9 shows the median $\Delta \log (M)$ has a narrow distribution at all luminosities, and is strongly peaked at $\Delta \log (M) \sim 0$. This indicates that the median of stellar masses for each galaxy, measured from all the methods in Table 3, successfully reproduces the input stellar mass. However, although this is the closest simulation to real data, the results here should be interpreted with caution as the simulations in TEST-2A are based on "free" input parameters in the fit (i.e., the SFH, population synthesis templates, metallicities, age and extinction were not fixed), the effect of which could be reflected on the median stellar mass (i.e., the input parameters are not the same among different methods, which could affect the estimated median values). Considering other independent results where the majority of the input parameters are fixed, as listed in Table 4 (and 2nd line in Table 7), one could assert that the median of the independently estimated stellar masses gives the closest agreement with the expected (input) mass.

Figures $10(\mathrm{a})$ and (b) present the relation between $\Delta \log (M)$ (from TEST-2A) and photometric $\mathrm{S} / \mathrm{N}$ and redshifts respectively. For most of the methods, an offset is present in $\Delta \log$ $(M)$ for high $\mathrm{S} / \mathrm{N}$, indicating that the errors in stellar masses are not necessarily caused by photometric uncertainties. There is an increase in the scatter at lower $\mathrm{S} / \mathrm{N}$ values (i.e fainter galaxies). Furthermore, we find a clear trend in $\Delta \log (M)$ as a function of redshift (Figure 10(b)), with some methods showing significantly larger scatter in $\Delta \log (M)$ at a given redshift. At higher redshifts, all the methods overestimate the stellar masses while the same methods underestimate the stellar mass for lower redshift galaxies. This is similar to result from Figure 7, where the stellar masses were underestimated at $M<10^{8} M_{\odot}$. The observed trend in Figure 10(b) is likely caused by a variety of different reasons. This is likely due to changes in the functional forms assumed for SFHs at different redshifts and the diversity of this parameter within the SAMs. For example, at high- $z$ almost all galaxies have rising SFHs while at low- $z$ there is a mix of quenched and star-forming galaxies. Furthermore, changes in extinction among galaxies, lower photometric S/N for some or re-cycling and mass loss could contribute to the observed trend.

The simulated templates based on the SAMs are generated from a diversity of SFHs (declining, increasing and constant) while the methods use simple prescriptions for the SFHs, causing an inconsistency in the mass estimation process. To explore if extinction is responsible for the observed trend and bimodality, we identify galaxies in Figure 10 (b) by their input $E(B-V)$ values. For method 1.A, high extinction $(E(B-V)>0.6)$ appears to be responsible for some of the observed bimodality but this is not the case for other methods. Methods that show bimodality in Figure 10(b) (1.A, 4.B and 10.G) use different population synthesis models (CB07 and M05) than the one used in the SAMs (BC03) from which the mock catalog is constructed. This introduces bias or additional errors in the mass estimate and hence, is responsible for the observed bimodality and the trend with redshift. This is particularly the case as the difference in the stellar mass estimates due to differences in the population synthesis codes (CB07, M05 and BC03) is dependent on redshift (see Section 6.2). However, method 6.C shows serious bimodality while using the same stellar synthesis model as the SAMs. Furthermore, since there is a change in the photometric $\mathrm{S} / \mathrm{N}$ with redshift, it is probable that photometric uncertainties is 
(a)
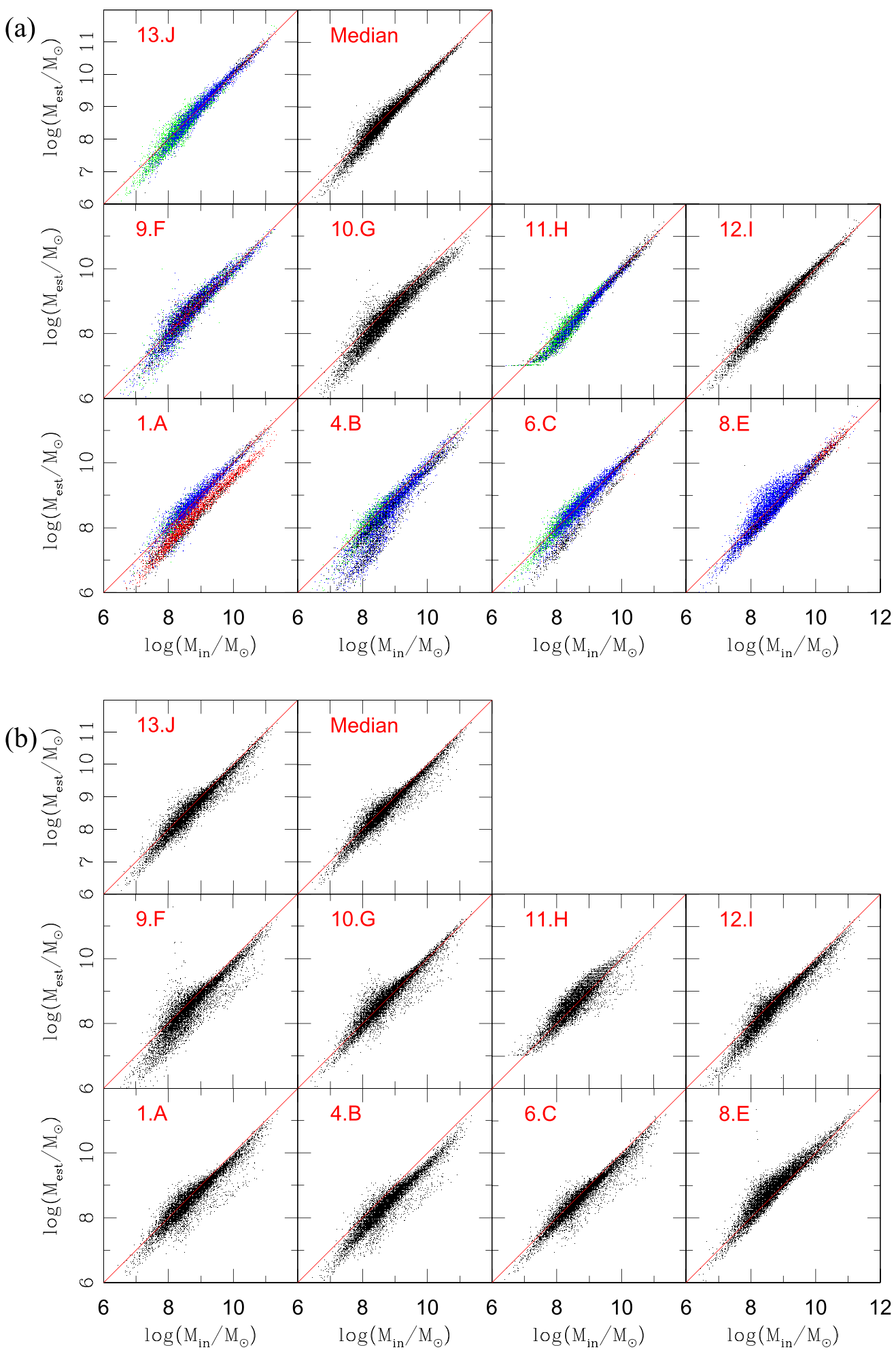

Figure 7. (a) Top: comparison between the input and estimated stellar masses for TEST-2A. The colors correspond to extinction associated with each galaxy, as estimated from the SED fits- $E_{(B-V)}=0$ (green); $0<E_{B-V}<0.3$ (blue); $0.3<E_{B-V}<0.6$ (black); $E_{B-V}>0.6$ (red). For the methods $10 . \mathrm{G}$ and $12 . \mathrm{I}$ no $E(B-V)$ values are available. There is a clear bi-modality in some cases. The red line corresponds to slope 1 . Most methods underestimate the stellar masses for galaxies with $M<10^{8} M_{\odot}$. (b) Bottom: comparison between the expected and estimated stellar masses for TEST-2B. The observed bi-modality in TEST-2A largely disappears when parameters are constrained. This test is designed to study the effects of free parameters on the estimated stellar mass by leaving all the parameters free (TEST-2A) and by constraining them (TEST-2B).

partly responsible for the observed trend in Figure 10(b).This is explored by restricting the sample in TEST-2A to galaxies with $\mathrm{S} / \mathrm{N}>10$. This does not remove the observed bimodality or the trend in the $\Delta(\log (M))-z$ relation, indicating that photometric errors are not responsible for the observed distribution of galaxies. 
Table 7

The rms Scatter, Bias and Outlier Fraction (OLF) in $\Delta \log (M)$ for TEST-2A (First Line) and TEST-2B (Second Line)

\begin{tabular}{lcccrc}
\hline \hline Code & rms & $\begin{array}{c}\text { rms } \\
\text { No Outliers }\end{array}$ & $\begin{array}{c}\text { rms } \\
{[2 \mathrm{~A}-2 \mathrm{~B}]}\end{array}$ & Bias & $\begin{array}{c}\text { Outlier } \\
\text { Fraction }\end{array}$ \\
\hline 1.A & 0.328 & 0.234 & 0.191 & 0.087 & 0.164 \\
& 0.267 & 0.201 & & 0.096 & 0.056 \\
4.B & 0.394 & 0.235 & 0.085 & 0.157 & 0.157 \\
& 0.403 & 0.314 & & 0.275 & 0.161 \\
6.C & 0.228 & 0.166 & 0.037 & 0.030 & 0.057 \\
& 0.225 & 0.177 & & 0.098 & 0.038 \\
7.D & 0.343 & 0.245 & 0.133 & 0.065 & 0.133 \\
& 0.368 & 0.224 & & 0.005 & 0.153 \\
8.E & 0.230 & 0.194 & 0.165 & 0.005 & 0.038 \\
& 0.283 & 0.223 & & -0.131 & 0.079 \\
9.F & 0.219 & 0.189 & 0.264 & 0.012 & 0.029 \\
& 0.343 & 0.220 & & 0.128 & 0.132 \\
10.G & 0.311 & 0.261 & 0.215 & 0.183 & 0.096 \\
& 0.225 & 0.170 & & -0.009 & 0.045 \\
11.H & 0.202 & 0.192 & 0.167 & 0.132 & 0.014 \\
& 0.279 & 0.200 & & 0.119 & 0.082 \\
12.I & 0.203 & 0.186 & 0.161 & 0.066 & 0.020 \\
& 0.259 & 0.222 & & 0.152 & 0.053 \\
13.J & 0.172 & 0.163 & 0.129 & 0.026 & 0.009 \\
& 0.215 & 0.187 & & 0.095 & 0.030 \\
Median & 0.175 & 0.168 & 0.110 & 0.069 & 0.008 \\
& 0.203 & 0.174 & & 0.068 & 0.028 \\
\hline
\end{tabular}

Note. $\operatorname{rms}[2 \mathrm{~A}-2 \mathrm{~B}]$ column gives the difference (in quadrature) between $\sigma$ values for TEST-2A and TEST-2B, defined as $\operatorname{rms}[2 \mathrm{~A}-2 \mathrm{~B}]=\sqrt{\sigma_{2 \mathrm{~A}}^{2}-\sigma_{2 \mathrm{~B}}^{2}}$. This quantifies contribution from free parameters to uncertainties in the stellar mass.

The observed filters refer to different rest-frame wavelengths and different redshift intervals. Therefore, the observed redshift dependence could be due to the fact that more of the light from shorter wavelengths (i.e., UV/optical light sensitive to SFR, reddening and age) is contributing to the observed light from high- $z$ galaxies while, the longer wavelengths (i.e., optical/ infrared light sensitive to stellar mass) are dominating the light for low- $z$ galaxies. This inherently introduces a redshiftdependent bias by weighting the fit toward different galaxy types. Pforr et al. (2012) showed that high- $z$ galaxies are easier to fit because the parameter space for degeneracies (specially age and dust) is more limited due to the small age of the universe at those redshifts. Using rest-frame $U-V$ colors, we divided the mock catalog into the red and blue galaxies and measured their respective stellar masses in redshift intervals. No significant difference was found between $\Delta(M)$ values from these two populations.

It is also important for the observed SEDs to cover the spectral breaks at any given redshift, as these breaks are essential for estimating physical parameters of galaxies. To quantify this, we identified the redshift interval where a certain break moves in or out of the observed wavelength range. We then measured and compared the median $\Delta(M)$ values for the two sets, separating galaxies in redshift bins to those with/ without the spectral features lying in that bin. If the observed redshift-dependence was due to this effect, we would expect to see a difference between the median $\Delta(M)$ values in redshift intervals. We find an average difference of only $0.03 \mathrm{dex}$ between the $(\Delta \log (M))$ values from the two samples, too small to be responsible for the observed trend by itself.

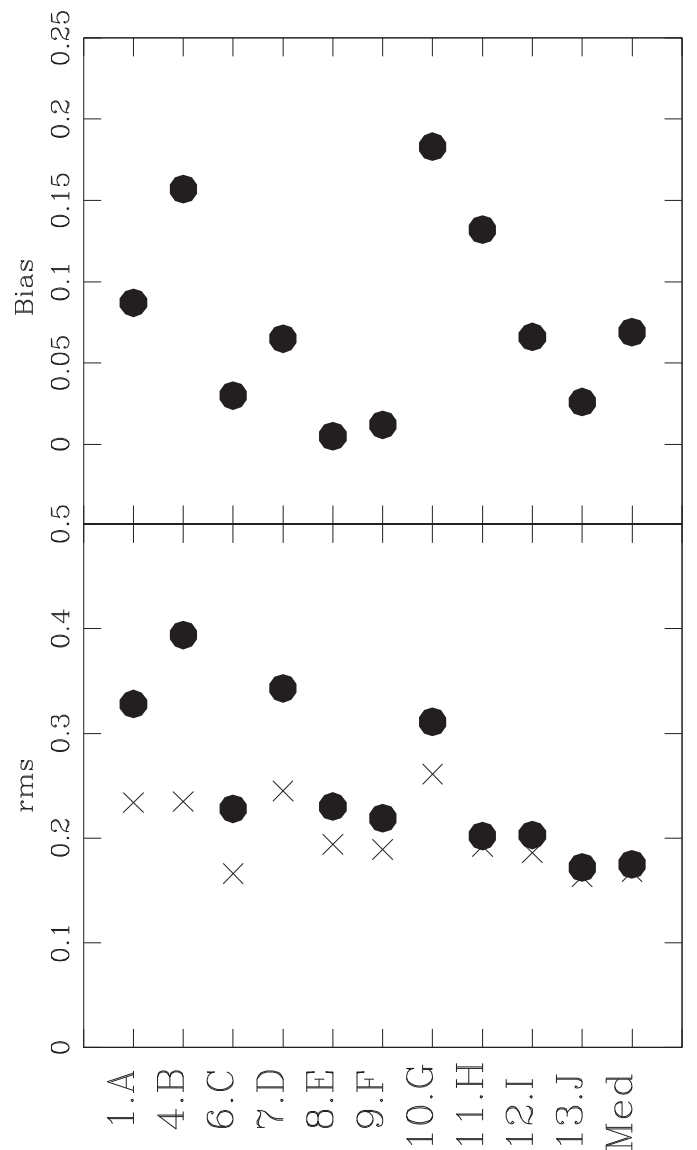

Figure 8. rms (bottom) and bias (top) in stellar masses measured from different codes are compared for TEST-2A. In the lower panel, filled circles represent rms estimates based on all the data while crosses are rms values with outliers excluded.

The effects of free parameters and in particular the population synthesis models are examined by studying the same relations using the data in TEST-2B, where all the teams used templates from $\mathrm{BC} 03$ (similar to the ones from which the mock catalogs are generated), zero extinction was assumed and the free parameters were all fixed (Table 1). The results are presented in Figures 11(a) and (b). The bi-modality observed for TEST-2A disappears however, the trend with redshift is still present.

As mentioned above, a possible cause of the observed trend in Figures 10(b) and 11(b) is different treatment of recycling and mass loss in the SAMs compared to the fitted models. The mock catalog here is generated using SAMs, which predict the multi-band photometry based on the $\mathrm{BC} 03$ model in the same way as the SED fitting codes, but predict stellar mass using the instantaneous recycling approximation, which does not accurately take into account the stellar mass loss as a function of time. In this scenario, the stellar mass is underpredicted at an early epoch after the stellar mass is formed, and overpredicted at a later epoch when the real stellar mass loss exceeds the adopted return fraction. We estimate that the change in the stellar mass due to instantaneous recycling is around 0.04 dex in $\Delta \log (M)$, with a clear trend with redshift. The expected trend due to recycling and mass loss is shown in Figure 11(b) (green boxes), indicating that it only plays a minor role in 

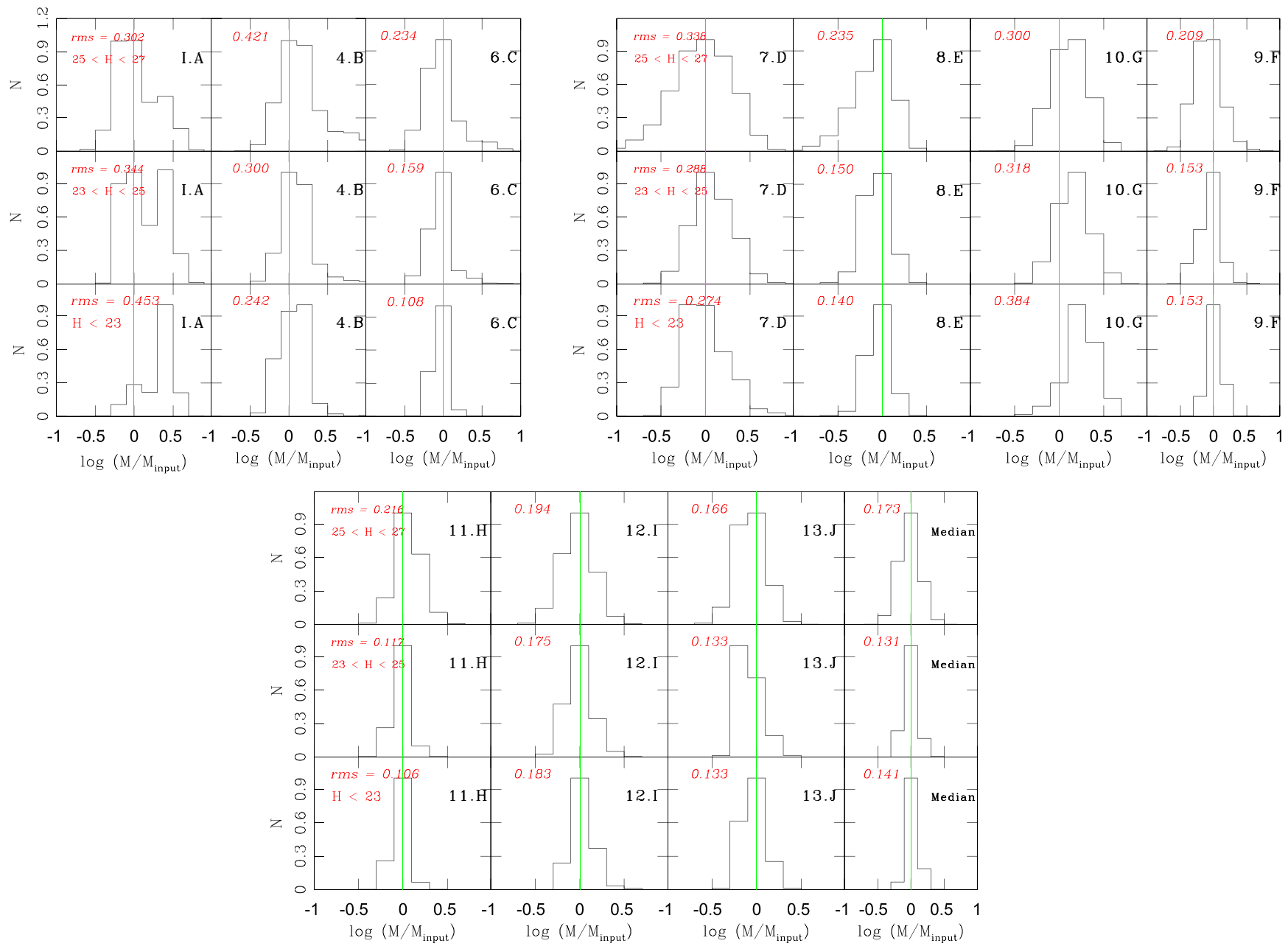

Figure 9. Histogram of $\Delta \log \left(M / M_{\text {input }}\right)$ values (from TEST-2A) in $H$-band magnitude intervals, estimated for each method separately. The rms values corresponding to each distribution are also shown. There is a clear increase in the width of the histogram toward fainter magnitudes. Also, there is a difference between the methods in terms of the spread in $\Delta \log \left(M / M_{\text {input }}\right)$. The median of the stellar masses provides narrow distribtions indicating that the median of different independent methods is a stable measure of the stellar mass.

explaining the observed trend. The conclusion is that although none of the effects, described above, could individually explain the observed trends in Figures $10(\mathrm{~b})$ and $11(\mathrm{~b})$, the combined contribution from the individual effects, could fully explain it.

In Figure 12 we compare the rms, bias and outlier fractions in $\Delta \log (M)$ between TEST-2A and TEST-2B. The rms values, even after removing the outliers, are still high $(\sim 0.2 \mathrm{dex})$. The green point in Figure 12 corresponds to the median mass. The observed scatter in the bias and outlier fractions between the two tests are identical, with methods that have higher rms scatter in TEST-2A also have high values in TEST-2B.

To explore the effect of photometric errors, for each galaxy in TEST-2A simulation we estimate the rms in $\Delta \log (M)$ between the values measured from different methods and plot it aganist the photometric $\mathrm{S} / \mathrm{N}$ in Figure 13. The scatter at any given $\mathrm{S} / \mathrm{N}$ indicates the $\mathrm{rms}$ in $\Delta \log (M)$ among different methods. As expected, there is significant scatter at lower $\mathrm{S} / \mathrm{N}$, with that decreasing toward higher values. The median rms in $\mathrm{S} / \mathrm{N}$ intervals are also shown in Figure 13. For TEST-2A, the rms distribution asymptotes around $\mathrm{rms} \sim 0.2 \mathrm{dex}$ at $\mathrm{S} / \mathrm{N}>20$. At these high $\mathrm{S} / \mathrm{N}$ values, the effect of photometric uncertainties on stellar mass measurement is negligible and all the scatter is due to systematic and code-dependent effects.
From TEST-1 we estimated that the contribution to the total rms due to method/code is $0.136 \mathrm{dex}$. Subtracting this, in quadrature, from the total rms for TEST-2A gives $\mathrm{rms}=0.146 \mathrm{dex}$, which is the rms scatter in stellar mass estimate, due to the effect of the free parameters. We carried out a linear fit to the median values in Figure 13 and find: $\mathrm{rms}=(-0.013 \pm 0.023) \mathrm{S} / \mathrm{N}+(0.409 \pm 0.230)$. Using this fit, one could estimate the rms values in the stellar mass for any given photometric $\mathrm{S} / \mathrm{N}$.

In conclusion, using realistic simulations from TEST-2A, we find the difference between the input (expected) and estimated masses $(\Delta(M))$ to follow a distribution that broadens from bright to faint magnitudes. At a given magnitude interval, while some methods show a relatively larger scatter in $\Delta(M)$, some show a systematic offset from the $\Delta(M)=0$ line. The observed offset in stellar mass is likely due to degenaracy between the free parameters (i.e., age and extinction). The offset is significantly reduced in TEST-2B where the input parameters are fixed. A trend was found between $\Delta(M)$ and redshift for both TEST-2A and TEST-2B. The most likely cause is the diversity of the SFHs used in the SAMs, from which the mock catalogs were constructed (and the fact that the methods mostly use simplified SFHs). 
The Astrophysical Journal, 808:101 (28pp), 2015 July 20

MOBASHER ET AL.

(a)

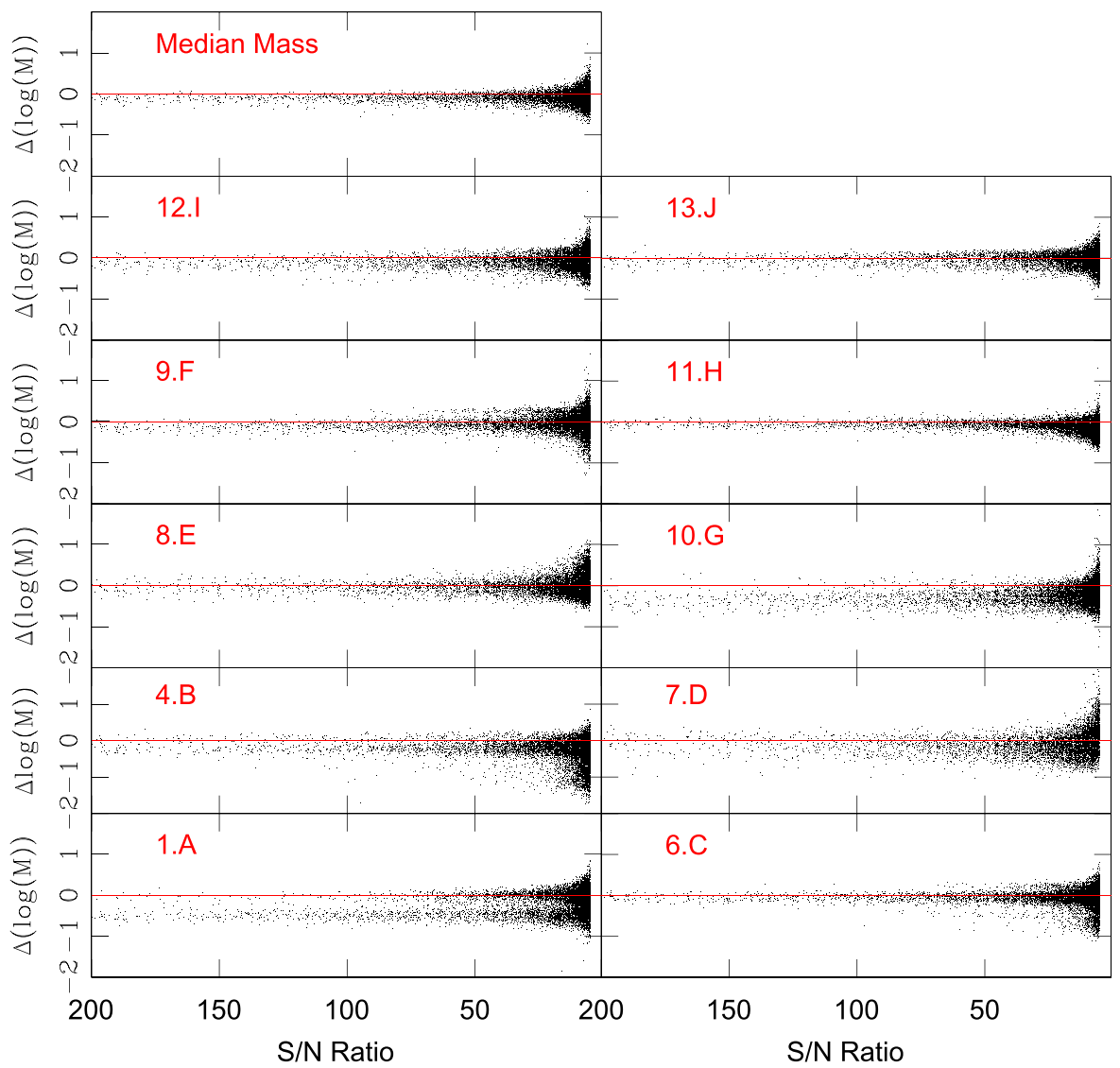

(b)

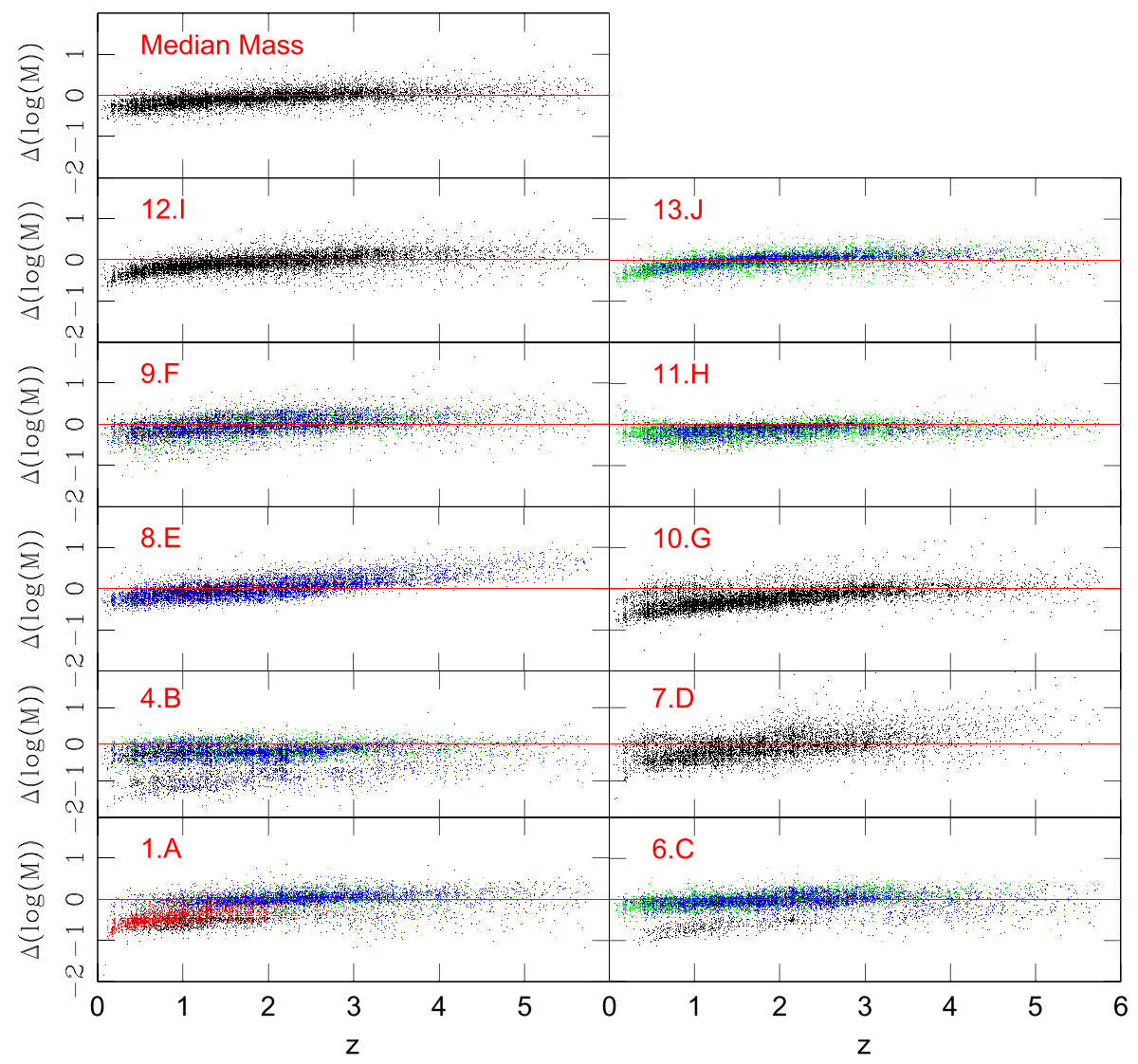

Figure 10. (a) Top panel: $\Delta \log (M)$ from TEST-2A as a function of the photometric S/N. (b) Bottom panel: $\Delta(\log ((M))$ from TEST-2A as a function of redshift. Colors correspond to different extinction values: $E_{(B-V)}=0$ (green); $0<E_{B-V}<0.3$ (blue); $0.3<E_{B-V}<0.6$ (black); $E_{B-V}>0.6$ (red). 

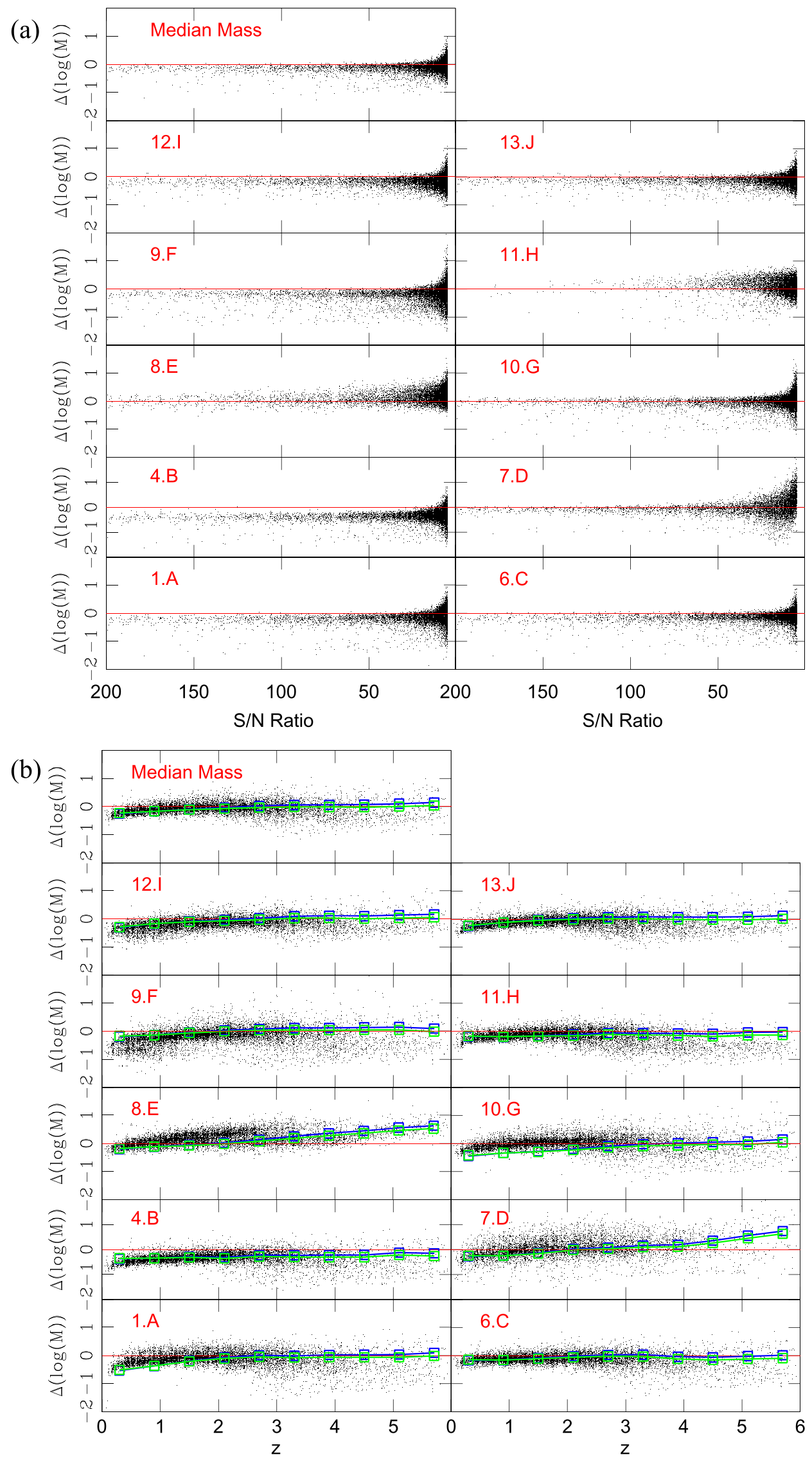

Figure 11. (a) Top panel: $\Delta(\log (M)$ from TEST-2B as a function of the photometric $\mathrm{S} / \mathrm{N}$. (b) Bottom panel: $\Delta \log (M)$ from TEST-2B is plotted as a function of redshift for TEST-2B. The blue boxes indicate the mean $\Delta \log (M)$ values in redshift bins based on TEST2-A (Figure $10(\mathrm{~b}))$. This shows changes in $\Delta \log (M)$ per redshift interval between TEST-2A and TEST-2B. The green boxes show the expected trend due to re-cycling and mass loss (see the text for details). The green line connects the green boxes. 

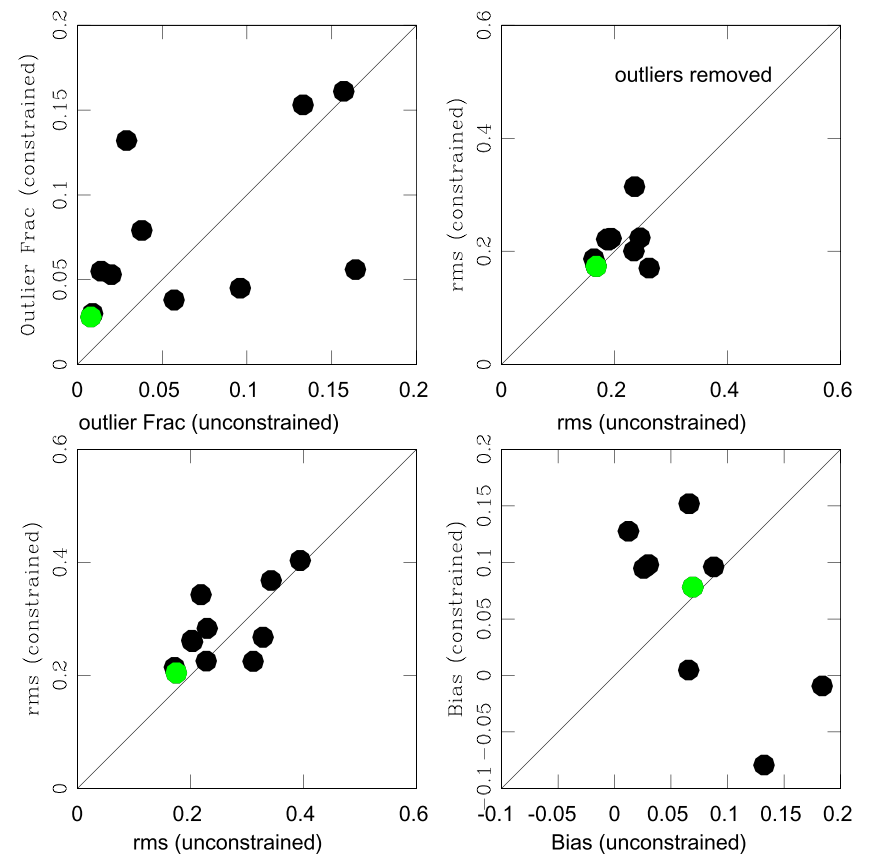

Figure 12. Comparison between the rms, bias and outlier fractions for TEST2A (horizontal) and TEST-2B (vertical). The green point corresponds to the median mass from all measurements.

\section{TEST-3: UNCERTAINTIES IN STELLAR MASS MEASUREMENT FROM OBSERVED DATA}

\subsection{Internal Tests of Stellar Mass Measurement Methods}

In this section we study the internal consistency in stellar mass estimates between different methods, using observed data (TEST-3). Unlike the mock catalogs, in case of the observational data we do not have prior knowledge of the expected stellar masses and therefore, this only provides an internal test of the consistency of mass measurements. Given this, we need to define a "reference" mass as a base to compare all the other masses with. Since the median mass is shown to be relatively unbiased (e.g., Figure 9), we adopt that as the "reference" mass. We note that the median of the stellar masses based on methods with different input parameters (TEST-3A) is not meaningful. Also, any bias in individual mass estimates would be reflected on to the median. However, this is only aimed to provide a relative test between different methods and the choice of the "reference" mass will not affect the results in this section. Furthermore, while a mass estimated from any other method here is equally acceptable as the "reference," it would still be susceptible to the above problems.

In Figure 14 we compare stellar masses predicted from different methods using TEST-3A and TEST-3B with the median mass, $M_{\text {med }} / M_{\odot}$, for each method. The rms, bias and outlier fractions in $\Delta \log \left(M_{\text {med }}\right)=\log \left(M_{\text {est }}\right)-\log \left(M_{\text {med }}\right)$ is estimated and listed in Table 8 . These should only be considered as relative measures, providing estimates of the overall agreement between masses from different methods and for individual methods between TEST-3A and TEST-3B. In case of TEST-3A, some methods show large scatter (e.g., 1.A and 4.B) and large outlier fraction (8.E) while others closely agree (11.H and 12.I). The behavior of these methods is consistent with results from the mock catalogs (TEST-2A). Also, the scatter between the estimated stellar masses from individual methods and the "reference" values are significantly reduced for most models when using TEST-3B (Figure 14 and Table 8). The sum of the rms values (in quadrature) in Table 8 gives the dispersion between different methods, corresponding to $0.39 \mathrm{dex}$ (for TEST-3A) and $0.22 \mathrm{dex}$ (for TEST-3B). The scatter in TEST-3A constitutes all the observational errors including photometric uncertainties and errors in the SED fitting process and hence, provides an estimate of the observed error associated with mass measurement from any given technique. The reduction in the rms scatter in TEST-3B is a result of the absence of constraints on the free parameters and the way different methods handle the SED fits.

Figure 15 examines the consistency of the stellar mass and extinction estimates between different methods. For any pair of methods, we find the difference between their estimated stellar mass $(\Delta(M))$ and extinction $(\Delta(\mathrm{ext}))$ values. Since these parameters are estimated simultaneously from the SED fits, this provides a direct and unbiased test of the consistency of the stellar mass and extinction estimates between different methods. It is clear that, in all cases, there is a shift on the $\Delta \log (M)-\Delta$ (ext) plane from $\Delta \log (M)=\Delta($ ext $)=0$ point for any of the two methods compared. Some methods agree on their estimated stellar mass and some on the extinction but none of the pair of methods agree in both.

\subsection{Dependence of the Stellar Mass on Population Synthesis Models}

The template SEDs generated by population synthesis models are the most fundamental components in measuring stellar mass of galaxies. It is therefore instructive to quantify the effect of the population synthesis models on the estimated mass of galaxies, given differences in the composition and data libraries used in these models. Here we estimate stellar masses using templates generated from $\mathrm{BC} 03$ and $\mathrm{CB} 07$ models while keeping all the rest of the parameters the same. The main difference between these two models is the addition of pulsating AGB stars to the CB07 model. For this experiment we use observational data from TEST-3, consisting of a sample of 586 galaxies. All the galaxies in this sample have spectroscopic data, used to fix redshifts of the galaxies when performing the SED fits. Since this is an internal comparison between results from the two models, there is no dependence on the "true" stellar mass values. The difference between the stellar masses using templates from BC03 and $\mathrm{CB} 07$ is plotted aganist redshift and mass in Figure 16, showing an offset of $\sim 0.2$ dex in $\log \left(M_{\mathrm{BC} 03} / M_{\mathrm{CB} 07}\right)$, with higher masses from the $\mathrm{BC} 03$. The difference between the stellar masses reduces at higher redshifts $(z>3)$ while it is constant over the entire stellar mass range studied here.

Given that the observational sample here is confined to brighter galaxies (for which spectroscopic data are available), it is possible that the above result is biased. To examine this, we apply the same procedure on the simulated data in TEST-2A (which is the most realistic). We find a similar shift $\sim 0.2 \mathrm{dex}$ in $\log \left(M_{\mathrm{BC} 03} / M_{\mathrm{CB} 07}\right)$ as for the observational data. The simulated galaxies also show closer agreement between the stellar masses at higher redshifts, in agreement with the result from TEST-3. The observed offset here is mainly due to the addition of the pulsating AGB stars to the CB07 model, affecting near-infrared part of the SEDs generated from it. At higher redshifts $(z \sim 3)$ the near-infrared light shifts outside the wavelength range spanned by the SEDs here and hence, the results (stellar masses) become unaffected by the AGB contribution, leading 

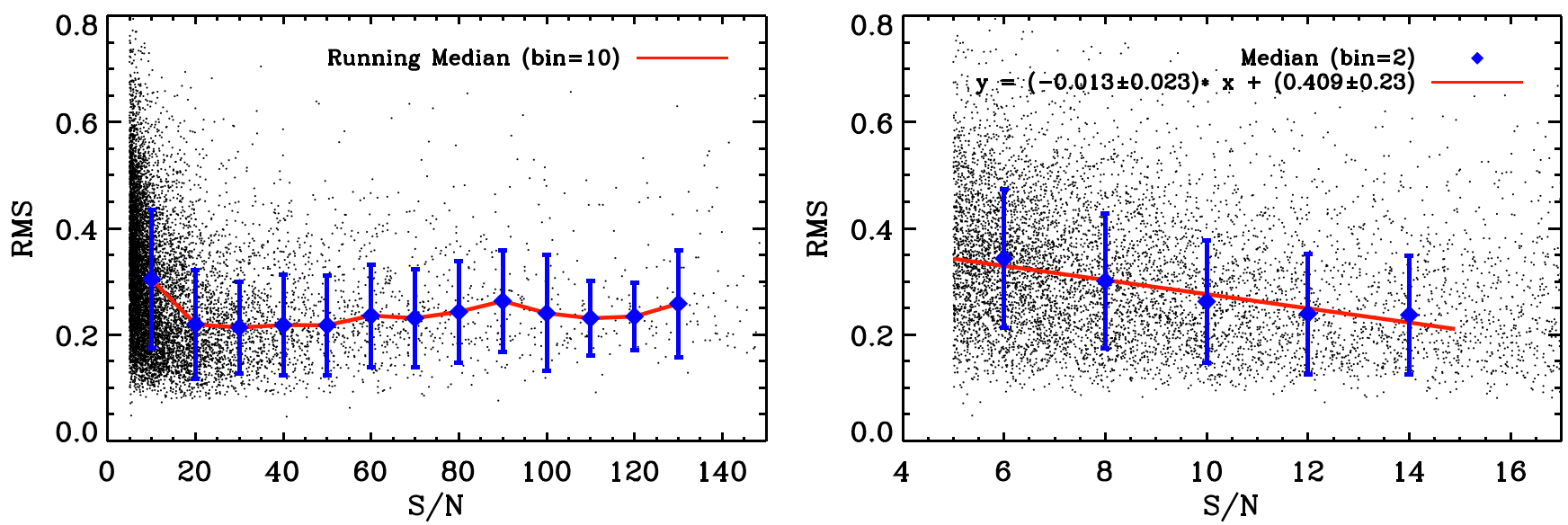

Figure 13. Left panel-the photometric $\mathrm{S} / \mathrm{N}$ are plotted vs. the rms in $\Delta \log (M)$ for each galaxy, measured from different methods. Right panel-the same as the left panel but plotted over a limited range of $\mathrm{S} / \mathrm{N}$ values. The boxes are the median $\Delta \log (M)$ values measured in $\mathrm{S} / \mathrm{N}$ intervals. The line is the least squares fit to the median points. The equation of the line can be used to estimate the rms values in stellar mass as a function of the S/N (see the text). The S/N corresponds to the $H$-band $(F 160 W)$ photometry.

to a better agreement in the estimated stellar mass between BC03 and CB07.

\subsection{Uncertainties in Stellar Mass Measurement due to Contributions from Nebular Emission}

It is well known that contribution from nebular emission lines is a non-negligible component of the observed flux from a galaxy at certain redshifts, leading to appreciable differences in the parameters estimated from their SEDs (Schenker et al. 2013a; de Barros et al. 2014). In the absence of correction for nebular emission, one overestimates both the stellar mass and age, as the nebular emission mimics an increase in the observed flux at longer wavelengths, enhancement of Balmer Breaks and hence, increased mass and age.

However, it is difficult to accurately quantify the effect of nebular emission in the estimated stellar mass in galaxies, as it depends on the redshift of the galaxy, the filters used for the SED fitting process and the width of the filters. For example, the $\mathrm{H} \alpha$ line shifts into the IRAC $3.6 \mu \mathrm{m}$ band at $z \sim 3.1$. Depending on the width of the filter, we get different fractional contributions to the observed fluxes. Therefore, the contribution due to nebular emission lines needs to be taken into account depending on the redshift of the galaxy in question and the filters used.

To quantify this, we estimated the stellar masses with and without correction for nebular emission lines, using the observed SEDs in TEST-3, keeping all the rest of the parameters fixed. We find a difference of up to $\sim 0.3 \mathrm{dex}$ in the estimated stellar mass, purely due to contribution from nebular emission lines.

In conclusion, differences in the stellar mass and extinction between differet methods when using observational data, confirm that the majority of the methods do not converge on the estimates of BOTH the stellar mass and extinction. Dependence of the stellar mass on population synthesis models was investigated and found that inclusion of pulsating AGB stars would decrease the estimate of the stellar mass by 0.2 dex. Finally, it was found that the contribution from nebular emission lines is to increase the stellar mass of galaxies by $\sim 0.3$ dex, depending on the redshift of the galaxy in question.

\section{TEST-4: THE EFFECT OF NEAR-INFRARED PHOTOMETRIC DEPTH AND SELECTION WAVELENGTH ON THE OVERALL STELLAR MASS}

To investigate the effect of near-infrared photometric depth on the estimated stellar mass, we designed TEST-4 which is similar to TEST-3A with the only difference being that it is based on a $z$-band selected sample (as compared to TEST-3A which was based on an $H$-band (F160W) selected sample) and with shallower near-infrared (JHK) data. Since this test also depends on the real data, we do not know the expected stellar masses. Using the median of the stellar masses measured for each galaxy by different methods, we estimate $\Delta \log \left(M_{\text {med }}\right)=$ $\log \left(M_{\text {est }}\right)-\log \left(M_{\text {Median }}\right)$ for individual galaxies. The rms in $\Delta \log \left(M_{\text {med }}\right)$ is then calculated for each method using all the galaxies, and for each galaxy using measurements from different methods. The results from TEST-3A and TEST4 are compared in Figure 17, which also presents comparison between the rms values when outliers are removed and between the bias estimates. There is no significant difference in the average mass estimates between the optical ( $z$-band) and nearIR ( $H$-band) selected samples. Also, no difference is found due to a relatively shallower near-IR photometry in TEST-4. Figure 18 presents the median rms values in $\mathrm{S} / \mathrm{N}$ bins for TEST-3A and TEST-4. There is no significant difference between the masses estimated from these two methods in terms of $\mathrm{S} / \mathrm{N}$, both converging to $\mathrm{rms}=0.2$ at $\mathrm{S} / \mathrm{N}>40$.

In conclusion, no significant difference is found in the estimated stellar masses due to the selection wavelength of the survey or the depth of the near-IR data alone.

\section{COMPARISON WITH OTHER STUDIES}

In recent years several studies have addressed the dependence of the stellar mass on physical parameters using simulated catalogs with known input values (Lee et al. 2009; Wuyts et al. 2009; Pforr et al. 2012). Longhetti \& Saracco (2009) studied the dependence of the estimated stellar masses on age, metallicity, IMF and SFH for early-type galaxies at $1<z<2$, using different stellar population synthesis codes to model their SEDs. They found that, at a given IMF, the stellar masses cannot be recovered better than a factor of 2-3. However, Michalowski et al. (2014) find a factor of $\sim 2$ scatter 

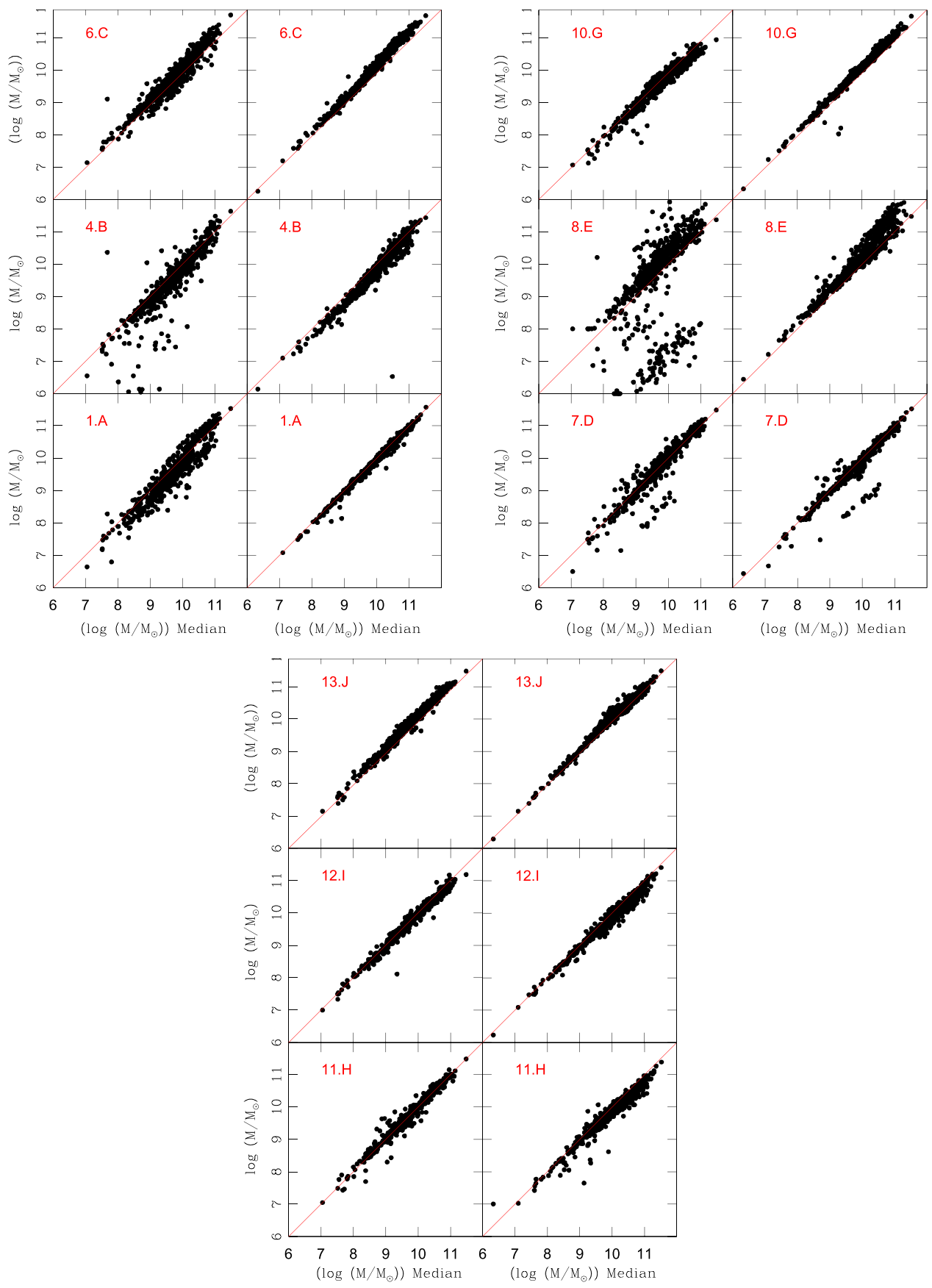

Figure 14. Relations between the median of stellar masses between all the methods and the estimated stellar mass from individual methods for both TEST-3A (left panels) and TEST-3B (right panels). There is a significant reduction in the scatter and outlier fraction in the case of TEST-3B.

in the stellar mass estimates solely due to the choice of the SFH.

Using model templates based on $\mathrm{BC} 03$, assuming Calzetti extinction law with reddening in the range $0-4$, and three SFHs: single stellar population (SSP), constant SFR and a $\tau$ model with $\tau=0.3 \mathrm{Gyr}$, Wuyts et al. (2009) generated mock catalogs in the redshift range $1.5<z<3$. When keeping redshift fixed, they underestimated the reddening, stellar mass and SFRs however, these estimates improved when redshift was used as a free parameter in the fit. While correctly predicting properties of spheroidal galaxies, they failed to reproduce input parameters for star-forming systems. Their results agree well with the independent study by Pforr et al. (2012).
Concentrating only on a simulated sample of Lyman Break Galaxies at $z \sim 3.4,4$ and 5, and using BC03, Lee et al. (2012) found that both masses and SFRs are underestimated while the ages are overestimated. They attributed this to differences in the SFHs between the mock and $\tau$-model templates used in the fitting process. They further showed that data spanning over a long wavelength range is essential to best recover the input parameters.

Pforr et al. (2012) performed a comprehensive study of uncertainties in the estimated physical parameters in galaxies. Based on the SED fitting code of Bolzonella et al. (2000) and population sythesis models from Maraston (2005), they found that the most important parameter in recovering the stellar mass 
Table 8

The rms Scatter, Bias and Outlier Fraction (OLF) in $\Delta \log \left(M_{\text {med }}\right)=\log \left(M_{\text {est }}\right)-\log \left(M_{\text {med }}\right)$ for different Methods Applied to TEST-3A (First Line) and TEST-3B (Second Line)

\begin{tabular}{llcrc}
\hline \hline Code & rms & $\begin{array}{c}\text { rms } \\
\text { Outliers Removed }\end{array}$ & Bias & $\begin{array}{c}\text { Outlier } \\
\text { Fraction }\end{array}$ \\
\hline 1.A & 0.327 & 0.256 & 0.111 & 0.108 \\
& 0.093 & 0.080 & 0.046 & 0.005 \\
4.B & 0.378 & 0.203 & 0.100 & 0.074 \\
& 0.247 & 0.230 & 0.171 & 0.028 \\
6.C & 0.223 & 0.206 & -0.040 & 0.014 \\
& 0.167 & 0.164 & -0.130 & 0.003 \\
7.D & 0.294 & 0.149 & -0.007 & 0.055 \\
& 0.268 & 0.114 & 0.043 & 0.044 \\
8.E & 0.938 & 0.297 & -0.237 & 0.345 \\
& 0.365 & 0.242 & -0.194 & 0.196 \\
10.G & 0.235 & 0.216 & 0.147 & 0.014 \\
& 0.143 & 0.125 & -0.101 & 0.003 \\
11.H & 0.132 & 0.106 & -0.003 & 0.014 \\
& 0.225 & 0.184 & 0.137 & 0.029 \\
12.I & 0.114 & 0.098 & 0.030 & 0.003 \\
& 0.183 & 0.175 & 0.128 & 0.010 \\
13.J & 0.146 & 0.146 & -0.110 & 0.00 \\
& 0.102 & 0.102 & -0.012 & 0.00 \\
\hline
\end{tabular}

Note. $M_{\text {med }}$ is the median of the stellar masses for a given galaxy, measured by different methods.

is the SFH, in agreement with Maraston et al. (2010). This underlines the importance of the physics of the model templates used in the SED fitting process. Using mock passive and starforming galaxies in redshift range $0.5<z<3$, they examined the sensitivity of the stellar mass to redshift. When spectroscopic redshifts are known, they find best stellar mass estimates at low redshift when reddening is excluded and at high redshift using reddening and inverted tau models (Pforr et al. 2012). The inclusion of reddening at low redshift causes severe underestimation for the stellar masses. When redshift is a free parameter in the fit (e.g., when no spec- $z$ are available), the additional degree of freedom allows for better mass estimates because redshift compensates for SFH and metallicity mismatch as well as the age-dust degeneracy (Pforr et al. 2013). This agrees well with results from the current study. At low redshifts, masses are still best determined excluding reddening from the fit.

In this paper we performed a critical study to quantify differences between the stellar masses estimated using different methods with model templates from different population synthesis codes, considering the existing degenaracies between the physical parameters. By fixing the physical parameters (specifically redshifts), we find a larger difference between the predicted and expected stellar masses. For example, by allowing the redshift to vary in the fit, it compensates for the mismatch between SFH and metallicity and age-dust degeneracy and hence, improves the recovery. In agreement with previous studies, we find that our lack of knowledge of the correct SFH, combined with inherent degeneracy between age, dust and metallicity, are the main reasons for uncertainties in stellar masses. Moreover, the estimated uncertainty depends on the wavelength coverage at any given redshift. We also investigated the effect of photometric uncertainties on these parameters and confirm that their effect is less serious than the above parameters.

\section{THE ERROR BUDGET}

In this section we quantify and compare relative contributions from the main sources dominating uncertainties in the stellar mass measurement. The situation becomes complicated by the fact that these parameters are correlated. Therefore, one needs to disentangle their individual contributions, as investigated by simulations in previous sections. In its general term, the uncertainty is defined as the rms scatter in $\Delta \log (M)=\log \left(M_{\text {input }}\right)-\log \left(M_{\text {est }}\right)$. The uncertainties in the stellar mass due to different parameters are listed in Table 9 and explained below:

Photometric errors: we examined this by estimating stellar masses for galaxies in mock catalogs (with known input mass) over a range of magnitudes. By using the same parameters to fit the SEDs as those used to generate the catalogs, we minimize the effect of other (free) parameters (TEST-1). Furthermore, by concentrating on individual codes, we avoid any codedependent effect (Table 3). Taking the above points into account, we estimate an uncertainty of $\sigma(\Delta \log (M))=0.134$ dex due to photometric errors. This dominates the error budget for galaxies with $m(160 W)>26$ (Figure 2(a)).

Codes/Methods: this was specifically tested by generating a simulated mock catalog (TEST-1) and constraining the input parameters, with the only free parameter being the code/method used. After subtracting the uncertainties due to photometric errors, we estimate the scatter in $\sigma(\Delta \log (M))$ among different codes in Table 4. We estimate an rms scatter of $\sigma(\Delta \log (M))=0.136$ dex due to differences in methods/ codes used.

Age and Extinction: in order to disentangle the effects of age and extinction and estimate their individual contribution to the error budget, we constructed a covarience matrix with $\sigma(\Delta \log (M))$ as matrix elements measured in different ageextinction grids. All the other variables were kept fixed. Results are listed in Table 6 and presented in Figure 5. The highest rms scatters were found for high extinction $\left(E_{B-V}>0.6\right)$ and age $\left(\sim 10^{9.5}\right)$ years values.

Numerical/Systematics: even if all the above uncertainties are accounted for, we still have an inherent "base" error, independent from photometric, code-dependent and the degeneracies mentioned above. We estimate this to be $\sigma(\Delta \log (M))=0.047$ dex (Figures 3 and 4 ).

Free Parameters: this is estimated by performing a realistic simulation where all the free parameters in the SED-fitting process were allowed to change (TEST-2A) and compared the results with a similar test where the parameters were kept fixed (TEST-2B). By subtracting (in quadrature) the rms estimates from TEST-2A and TEST-2B, we find a scatter among different methods, (due to free parameters), in the range $\sigma(\Delta \log (M))=0.037-0.264$ dex (Table 7). The rms scatter associated with free parameters from the median stellar mass values (from TEST-3A) is 0.110 dex, which is taken as our estimate of the uncertainty in the stellar mass measurement caused by free parameters.

Combined Observational Uncertainties: using the observed data (TEST-3A), we measure the scatter in the estimated stellar mass values among different codes. This is estimated to be $\sigma(\Delta \log (M))=0.390$ dex.

Selection Wavelength and Photometric Depth: TEST-4 was formulated to address this and predicts a contribution $<0.2$ dex in the total error budget due to the selection of the wavelength and photometric depth of the sample. 

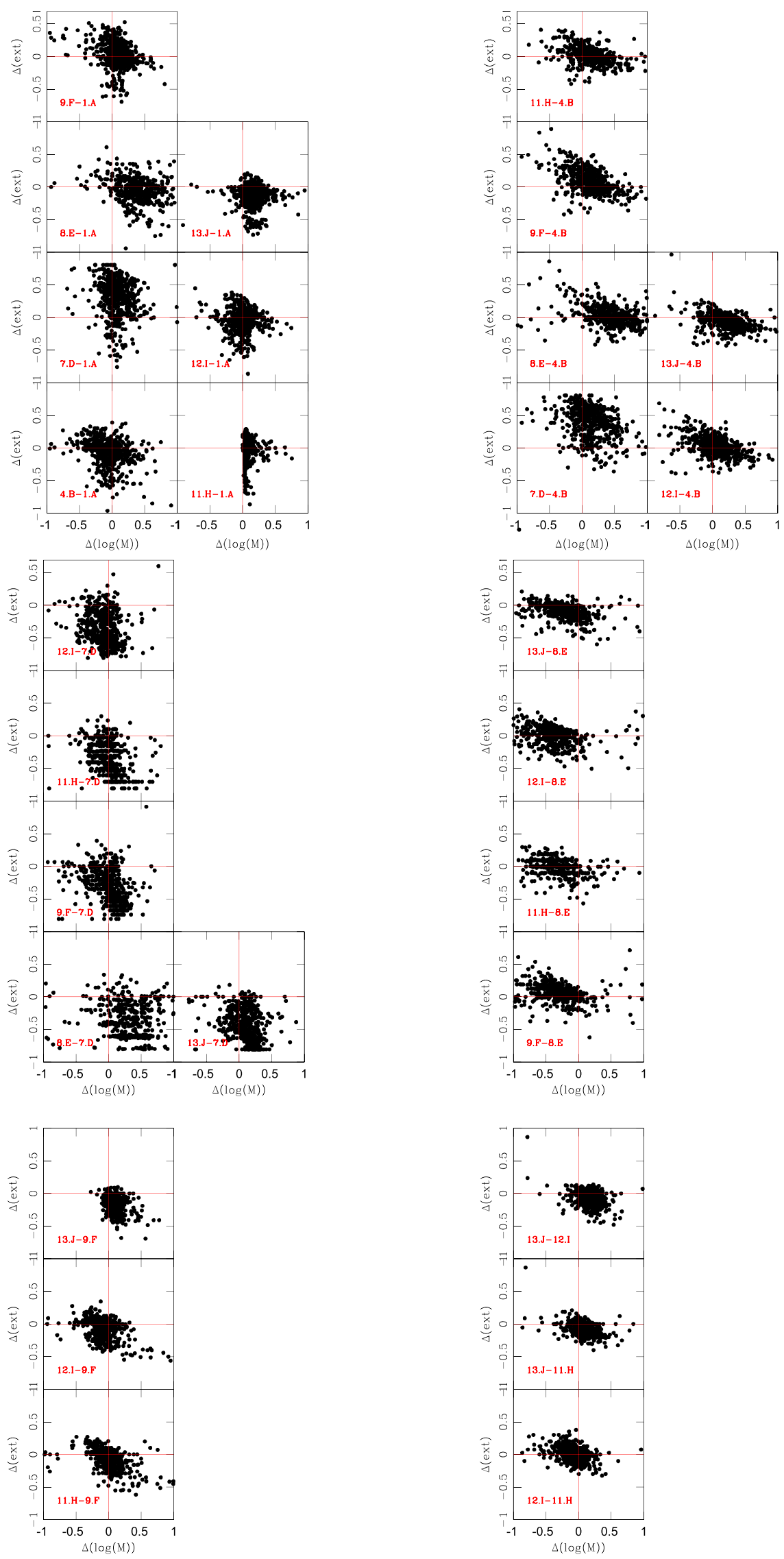

Figure 15. Compares the difference in the stellar mass and extinction $-\Delta \log (M)$ and $\Delta$ (ext) respectively—between any two methods using TEST-3A applied on 586 observed galaxies in GOODS-S. The scatter in these diagrams around the center $(\Delta \log (M)=\Delta($ ext $)=0)$ indicates that stellar mass measurement methods cannot at the same time consistently produce both the stellar mass and extinction. 

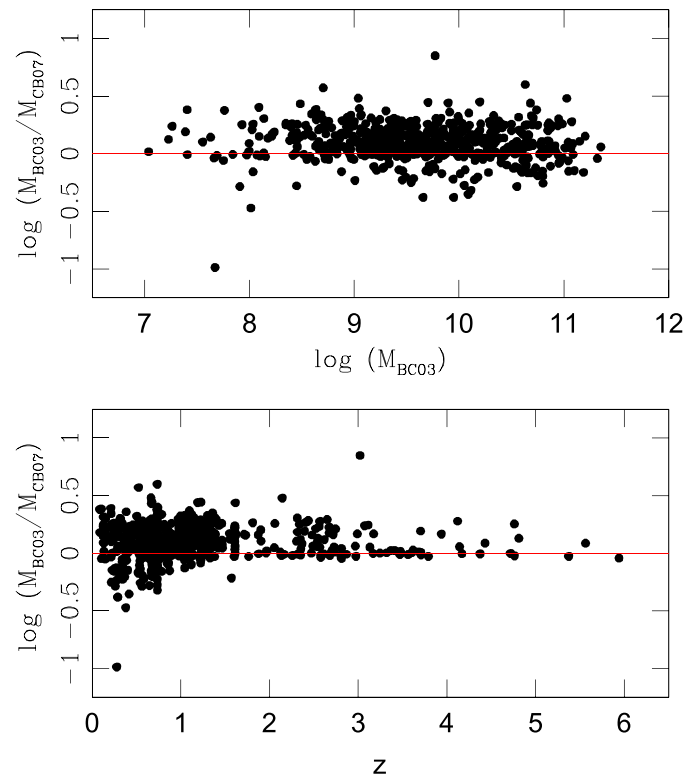

Figure 16. Comparison between the stellar mass estimates based on the $\mathrm{BC} 03$ and CB07 population synthesis models using observational data from TEST-3. All the parameters in the SED fits are fixed, with the only difference being the population synthesis models which generate the template SEDs.
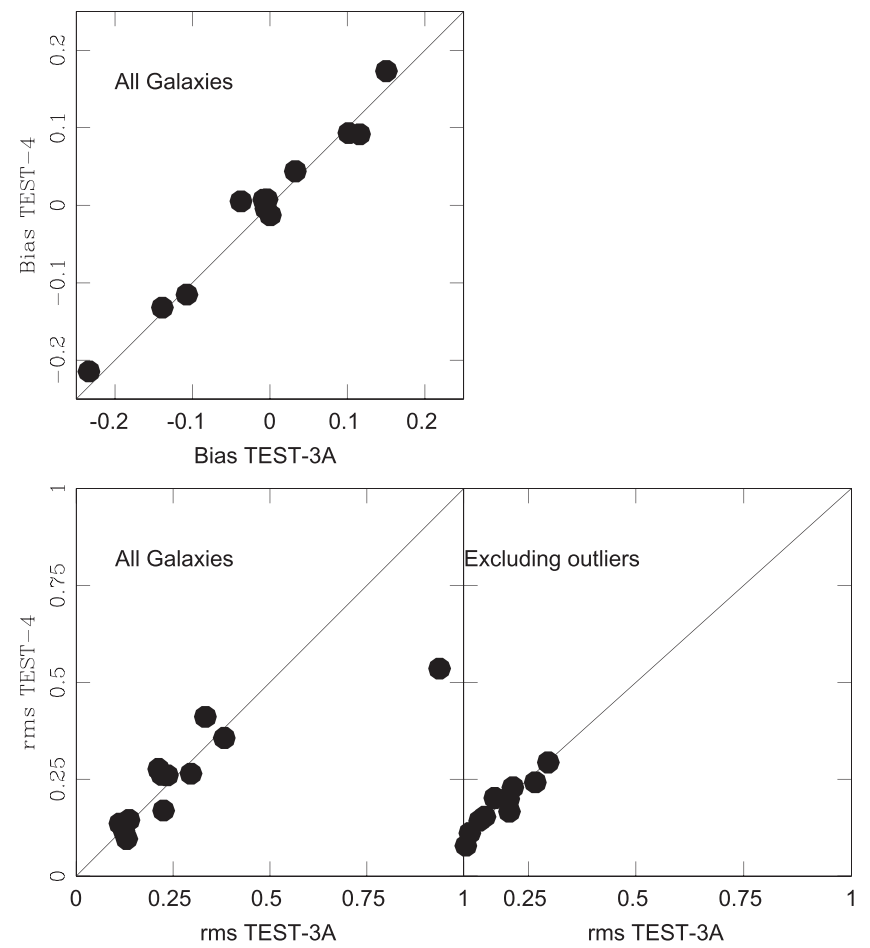

Figure 17. Compares the rms values in $\Delta \log \left(M_{\text {Med }}\right)=\log \left(M_{\text {est }}\right)-$ $\log \left(M_{\text {Med }}\right)$, measured from different codes, between TEST-3A and TEST-4. $M_{\text {med }}$ is the median of the mass estimates for individual galaxies from different codes. Also, presented are the comparison between the rms values with the outliers removed and the bias resulted from different methods.

Nebular Line Correction: in TEST-3 (based on the observational data), we compared the stellar mass estimates with and without correction for nebular emission (both line and continuum). We estimate an average error of $0.5 \mathrm{dex}$ in the stellar masses due to contribution from nebular line emission.

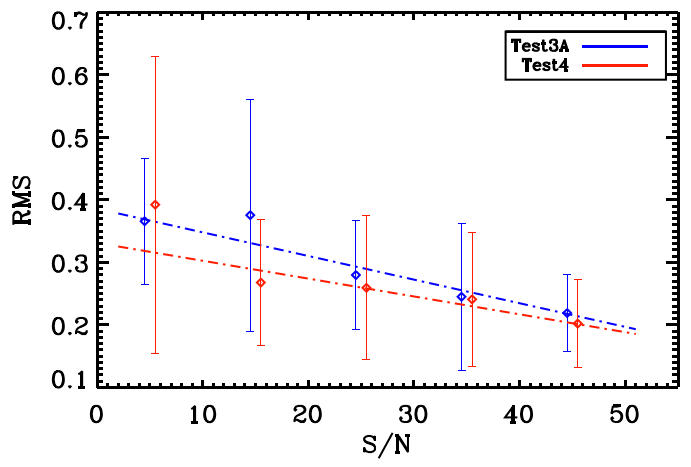

Figure 18. Median of the rms values in $\Delta \log \left(M_{\text {med }}\right)=\log \left(M_{\text {est }}\right)-\log \left(M_{\text {Med }}\right)$ estimated in S/N intervals for TEST-3 and TEST- $4 . M_{\text {med }}$ is the median of the mass estimates for individual galaxies from different codes. The plot shows results for both TEST-3A and TEST-4. The lines are the best fits to the median values.

Table 9

Error Budget for Stellar Mass Measurement

\begin{tabular}{ll}
\hline \hline & $\operatorname{rms}$ in $\log \left(M / M_{\odot}\right)$ \\
Method & 0.136 \\
Systematic & 0.050 \\
Photometry & 0.135 \\
Numerical & 0.045 \\
$\log ($ Age $)$ & $E_{(B-V)}$ \\
& $(<0.3,0.3-0.6,>0.6)$ \\
$7-8$ & $(0.121,0.141,0.307)$ \\
$8-9$ & $(0.112,0.180,0.297)$ \\
$9-10$ & $(0.273,0.271,0.387)$ \\
Free Parameters & 0.110 \\
Nebular lines & 0.300 \\
Combined Observational & 0.390 \\
Population Synthesis Models & 0.200 \\
Depth (near-IR photometry) & $<0.200$ \\
\hline
\end{tabular}

Population Synthesis Models: the templates used to measure stellar masses were generated by population synthesis models. We studied the effect of pulsating AGB stars on these templates and on the resulting stellar mass and find this to change the stellar mass by $\sim 0.2$ dex.

\section{SUMMARY AND CONCLUSIONS}

We performed a detailed study of the errors and main sources of uncertainty in stellar mass measurement in galaxies. Generating simulated galaxy catalogs with known input parameters (redshift, mass, SEDs), we investigated deviations in the estimated stellar mass from their input values $(\Delta \log (M))$ and its dependence on the observable parameters. The stellar masses were measured by 10 independent methods/codes with the results compared. Conclusions from this study are summarized below.

1. When the same set of input assumptions are used, no significant bias is found between different methods. We find that the spread in the stellar mass of any given galaxy, using different methods is $\sigma(\Delta \log (M))=0.136$ dex. Fainter galaxies with lower photometric $\mathrm{S} / \mathrm{N}$ $(H>26 \mathrm{mag})$ are responsible for most of this scatter.

2. When the same population synthesis models and parameters are used, the median of the stellar masses from different methods provides the smallest rms scatter 
(with respect to the input stellar mass values) compared to individual methods.

3. We separated degeneracies in stellar mass measurements due to age and extinction and estimated their individual contribution to the total error budget. We find that the rms in stellar mass significantly increases for $E_{B-V}>0.6$ for all ages. For any given method and extinction, there is an increase in the estimated stellar mass for ages $>10^{8.5}$ years.

4. From our simulations we found that errors in the stellar mass and age are strongly correlated (galaxies with large deviations in their stellar mass also show large deviations in age). A weaker trend is found with the extinction.

5. The effect of free parameters on stellar mass estimates was studied using mock photometric catalogs with known input stellar mass. We find $\sigma(\Delta \log (M))=0.136 \mathrm{dex}$, caused by degeneracy and interplay between parameters.

6. The effects of population synthesis models and corrction for nebular emission were investigated and found to change the stellar mass $(\Delta(\log (M)))$ by 0.2 and 0.3 dex respectively.

\section{APPENDIX A \\ DEFINITIONS OF STELLAR MASS IN GALAXIES}

There are three different definitions of the stellar mass commonly used in literature.

1. Stellar mass is built up over time by star formation activity in galaxies, with stellar mass recycling ignored. If $\phi(t) d t$ is the stellar mass generated in a galaxy between time $t$ and $t+\Delta t$ with a star formation rate $\phi(t)$, the stellar mass over the age of the galaxy is $M_{\mathrm{int}}=\int_{0}^{t_{\mathrm{g}}} \phi(t) d t$, where $t_{\mathrm{g}}$ is the current age of the galaxy. In this case the stellar mass depends on the SFH of the galaxy. Assuming an exponentially declining SFR, $\operatorname{SFR}(t)=\operatorname{SFR}_{0} e^{-t / \tau}$, the stellar mass is therefore calculated for each object as

$$
M_{\mathrm{int}}=\tau \operatorname{SFR}_{0}\left(e^{t / \tau}-1\right)
$$

where $\mathrm{SFR}_{0}$ is the SFR at $t=0$ and $\tau$ is the SFR time scale.

2. Stellar mass recycling is taken into account using the "instantaneous recycling approximation." In this case a fixed fraction of the mass that goes into stars is returned to the interstellar medium (ISM) immediately in each timestep to take into account the stellar mass loss in supernovae explosion or stellar winds. At any given time interval, $\Delta t$, the increment of the stellar mass is the star formation rate minus the mass fraction of the short-lived stars and stellar winds multiplied by the time interval. Therefore, the stellar mass of a galaxy at the age of $t_{\mathrm{g}}$, assuming instantaneous recycling approximation, $M_{\text {ira }}$ is estimated as

$$
M_{\text {ira }}=\int_{0}^{t_{\mathrm{g}}}\left(\phi(t)-\phi(t) R_{\mathrm{re}}\right) d t=\left(1-R_{\mathrm{re}}\right) \int_{0}^{t_{\mathrm{g}}} \phi(t) d t
$$

where $\phi(t)$ is the SFR at time $t$ and $R_{\text {re }}$ is the recycling fraction, which is set to be a constant and depends on the IMF. Most SAMs adopt this prescription.

3. Stellar mass recycling is treated using detailed predictions of stellar population models for how much mass is returned from a stellar population of a given age in each timestep (e.g., Lu et al. 2015). The stellar mass of a galaxy at the current age $t_{\mathrm{g}}$ depends on the SFH and the mass loss from all stars formed
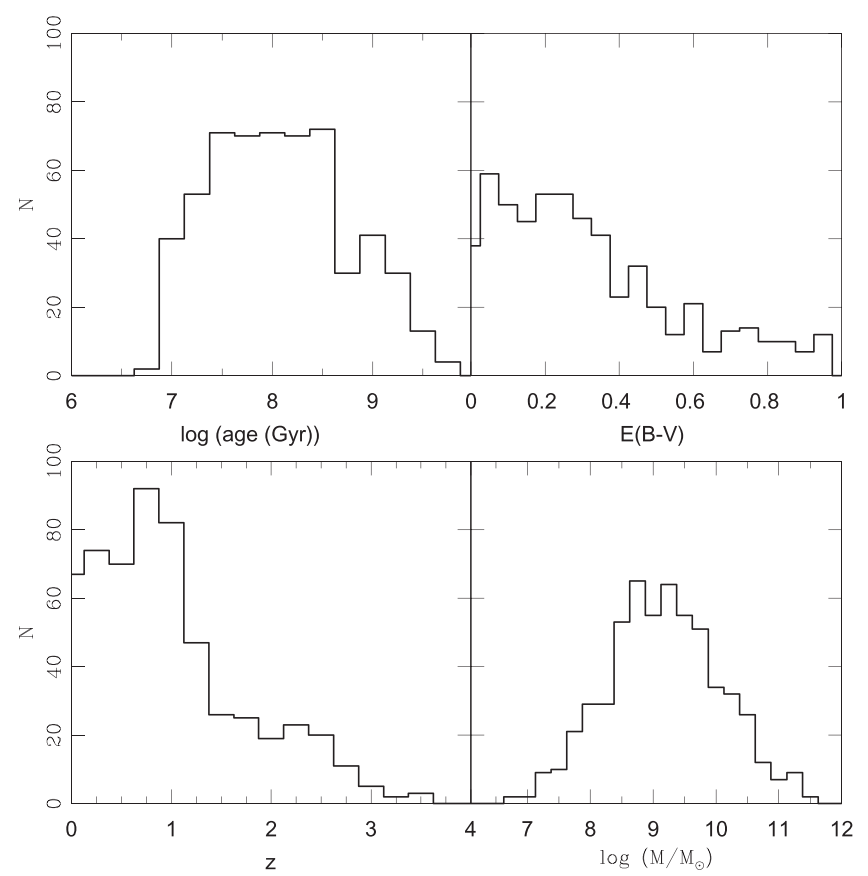

Figure 19. Distribution of physical parameters (redshift, stellar mass, age and extinction) for the TEST-1 mock catalog. The redshift distribution is taken to be the same as the observed distribution in the spectroscopic sample used for training the mock catalog.

in the past and is estimated as

$$
M_{*}=\int_{0}^{t_{\mathrm{g}}}\left[\phi(t)-\phi(t) R_{\mathrm{re}}\left(t_{\mathrm{g}}-t\right)\right] d t
$$

where $R_{\mathrm{re}}\left(t_{\mathrm{g}}-t\right)$ is the recycling fraction at time $t_{\mathrm{g}}$ for the stellar mass formed at time $t$. The stellar mass of galaxies strongly depends on their SFH, with the recycled mass mainly depending on the IMF and age, with a secondary dependence on the metallicity of the stellar population. We show in Section 6 that the stellar mass of galaxies only weakly depends on the stellar mass loss.

\section{APPENDIX B \\ TEST-1 AND TEST-2 SIMULATED CATALOGS}

To generate the mock catalog to be as close as possible to the observed data, we first predict the observed one-dimensional distributions of the expectation values for each of the main parameters (redshift, age, stellar mass and extinction). This is done by using a sample of galaxies in GOODS-S with available spectroscopic redshifts and by fitting their SEDs to model templates generated from $\mathrm{BC} 03$. For each parameter, we generated the one-dimensional distribution for the observed parameters and fitted them to analytic functions (i.e., Gaussian). We then drew a mock sample of 1000 galaxies from this distribution (with their associated multi-waveband photometry) and only retained those with (a) ages between $10 \mathrm{Myr}$ and the age of the universe at the redshift of the galaxy and (b) with $0<E(B-V)<1$. The final sample selected for TEST-1 mock catalog satisfies these criteria, with the total number of galaxies adjusted to be similar to the spectroscopic sample in GOODS-S. This test therefore contains 559 simulated points. The distribution of the main parameters in TEST-1 catalog are presented in Figure 19. The redshift 


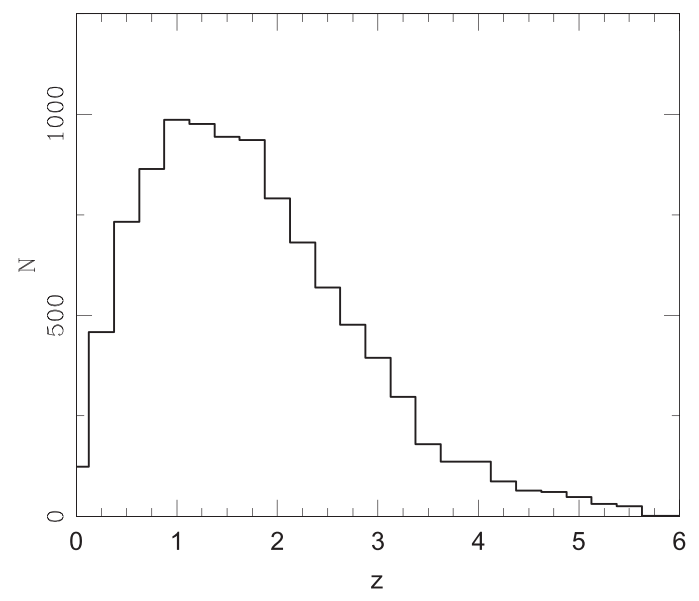

Figure 20. Input redshift distribution for TEST-2A mock catalog.

distribution here closely resembles the observed distribution for galaxies with spectroscopic redshifts in the GOODS-S field.

While a larger mock catalog (in terms of the number of galaxies generated) would reduce the poisson noise in the analysis, we aimed for a catalog which contains similar number of galaxies as those in the observed spectroscopic catalog. This allows a more realistic estimate of the stellar mass calibration errors when applying the results from the mock data to the real data.

For TEST-2, light cones were used to directly replicate CANDELS field geometry. The $N(z)$ for this model is generated to closely resemble the photometric redshift distribution for the GOODS-S field, as shown in Figure 20. In this test, stellar mass recycling is treated using the "instantaneous" recycling approximation in which a fixed fraction of mass that goes into stars is immediately returned into the ISM during each time step (Lu et al. 2015).

\section{REFERENCES}

Acquaviva, V., Gawiser, E., Bickerton, S. J., et al. 2012, ApJ, 749, 72 Acquaviva, V., Gawiser, E., \& Guaita, L. 2011, ApJ, 737, 47
Arnouts, S., \& Ilbert, O. 2011, ascl soft, ascl:1108.009

Ashby, M. L. N., Willner, S. P., Fazio, G. G., et al. 2013, ApJ, 769, 80

Beckwith, S. V. W., Stiavelli, M., Koekemoer, A. M., et al. 2006, AJ, 132, 1729

Behroozi, P. S., Wechsler, R. H., Wu, H.-Y., et al. 2013, ApJ, 763, 18

Bolzonella, M., Miralles, J.-M., \& Pelló, R. 2000, A\&A, 363, 476

Bouwens, R. J., Illingworth, G. D., Oesch, P. A., et al. 2011, ApJ, 737, 90

Brammer, G. B., van Dokkum, P. G., \& Coppi, P. 2008, ApJ, 686, 1503

Bruzual, G., \& Charlot, S. 2003, MNRAS, 344, 1000

Charlot, S., \& Fall, S. M. 2000, ApJ, 539, 718

Dahlen, T., Mobasher, B., Faber, S. M., et al. 2013, ApJ, 775, 93

de Barros, S., Schaerer, D., \& Stark, D. P. 2014, A\&A, 563, A81

Finkelstein, S. L., Papovich, C., Ryan, R. E., et al. 2012, ApJ, 758, 93

Giavalisco, M., Ferguson, H. C., Koekemoer, A. M., et al. 2004, ApJL, 600, L93

Grogin, N. A., Kocevski, D. D., Faber, S. M., et al. 2011, ApJS, 197, 35

Guo, Y., Ferguson, H. C., Giavalisco, M., et al. 2013, ApJS, 207, 24

Johnson, S. 2013, ascl soft, ascl:1309.005

Klypin, A. A., Trujillo-Gomez, S., \& Primack, J. 2011, ApJ, 740, 102

Koekemoer, A. M., Faber, S. M., Ferguson, H. C., et al. 2011, ApJS, 197, 36

Kriek, M., van Dokkum, P. G., Labbé, I., et al. 2009, ApJ, 700, 221

Laidler, V. G., Papovich, C., Grogin, N. A., et al. 2007, PASP, 119, 1325

Lee, S.-K., Ferguson, H. C., Somerville, R. S., et al. 2014, ApJ, 783, 81

Lee, K.-S., Ferguson, H. C., Wiklind, T., et al. 2012, ApJ, 752, 66

Lee, S.-K., Idzi, R., Ferguson, H. C., et al. 2009, ApJS, 184, 100

Longhetti, M., \& Saracco, P. 2009, MNRAS, 394, 774

Lu, Y., Mo, H. J., \& Wechsler, R. H. 2015, MNRAS, 446, 1907

Maraston, C. 2005, MNRAS, 362, 799

Maraston, C., Pforr, J., Renzini, A., et al. 2010, MNRAS, 407, 830

McLure, R. J., Dunlop, J. S., Bowler, R. A. A., et al. 2013, MNRAS, 432, 2696

Michalowski, M. J., Hayward, C. C., Dunlop, J. S., et al. 2014, A\&A, 571, A75

Oke, J. B., \& Gunn, J. E. 1983, ApJ, 266, 713

Ouchi, M., Mobasher, B., Shimasaku, K., et al. 2009, ApJ, 706, 1136

Pforr, J., Maraston, C., \& Tonini, C. 2012, MNRAS, 422, 3285

Pforr, J., Maraston, C., \& Tonini, C. 2013, MNRAS, 435, 1389

Reddy, N. A., Pettini, M., Steidel, C. C., et al. 2012, ApJ, 754, 25

Santini, P., Ferguson, H. C., Fontana, A., et al. 2015, ApJ, 801, 97

Schenker, M. A., Ellis, R. S., Konidaris, N. P., \& Stark, D. P. 2013a, ApJ, 777,67

Schenker, M. A., Robertson, B. E., Ellis, R. S., et al. 2013b, ApJ, 768, 196

Scoville, N., Abraham, R. G., Aussel, H., et al. 2007, ApJS, 172, 38

Somerville, R. S., Gilmore, R. C., Primack, J. R., \& Domínguez, A. 2012, MNRAS, 423, 1992

Somerville, R. S., Hopkins, P. F., Cox, T. J., Robertson, B. E., \& Hernquist, L. 2008, MNRAS, 391, 481

Wiklind, T., Dickinson, M., Ferguson, H. C., et al. 2008, ApJ, 676, 781

Wuyts, S., Franx, M., Cox, T. J., et al. 2009, ApJ, 700, 799 